\title{
The effect of Ni on element partitioning during iron meteorite crystallization
}

\author{
Nancy L. CHABOT ${ }^{1 *}$, Sarah A. SASLOW ${ }^{2}$, William F. McDONOUGH ${ }^{3}$, and Timothy J. McCOY ${ }^{4}$ \\ ${ }^{1}$ Applied Physics Laboratory, 11100 Johns Hopkins Road, Laurel, Maryland, 20723, USA \\ ${ }^{2}$ University of Maryland, College Park, Maryland, 20742, USA \\ ${ }^{3}$ Department of Geology, University of Maryland, College Park, Maryland, 20742, USA \\ ${ }^{4}$ Department of Mineral Sciences, National Museum of Natural History, Smithsonian Institution, Washington, D.C., 20560, USA
}

*Corresponding author. E-mail: nancy.chabot@jhuapl.edu

(Received 02 January 2006; revision accepted 29 April 2007)

\begin{abstract}
Iron meteorites exhibit a large range in Ni concentrations, from only $4 \%$ to nearly $60 \%$. Most previous experiments aimed at understanding the crystallization of iron meteorites have been conducted in systems with about $10 \% \mathrm{Ni}$ or less. We performed solid metal/liquid metal experiments to determine the effect of $\mathrm{Ni}$ on partition coefficients for 20 trace elements pertinent to iron meteorites. Experiments were conducted in both the end-member Ni-S system as well as in the Fe-Ni$\mathrm{S}$ system with intermediate Ni compositions applicable to high-Ni iron meteorites. The Ni content of the system affects solid metal/liquid metal partitioning behavior. For a given $\mathrm{S}$ concentration, partition coefficients in the Ni-S system can be over an order of magnitude larger than in the Fe-S system. However, for compositions relevant to even the most Ni-rich iron meteorites, the effect of Ni on partitioning behavior is minor, amounting to less than a factor of two for the majority of trace elements studied. Any effect of Ni also appears minor when it is compared to the large influence $\mathrm{S}$ has on element partitioning behavior. Thus, we conclude that in the presence of an evolving S-bearing metallic melt, crystallization models can safely neglect effects from $\mathrm{Ni}$ when considering the full range of iron meteorite compositions.
\end{abstract}

\section{INTRODUCTION}

Every iron meteorite is classified as belonging to a "magmatic" group, a "nonmagmatic" group, or as being "ungrouped." Magmatic groups are believed to sample the central metallic cores of asteroid-sized bodies, with each group representing a separate asteroidal core (e.g., Scott 1972). In contrast, the iron meteorites of nonmagmatic groups commonly contain silicate inclusions and do not exhibit the characteristic large trace element fractionations that are present within magmatic groups, suggesting a more complex history (e.g., Scott and Wasson 1975). Although we adopt the term "nonmagmatic" to refer to these groups, an origin involving crystallization from a metallic melt is indicated for all iron meteorites, including the nonmagmatic ones. Currently, just over 12\% (Chabot and Haack 2006) of all iron meteorites do not have at least four other irons to which they appear genetically related, leaving them classified as ungrouped iron meteorites. If these ungrouped irons are not related to the current groups, the ungrouped irons may represent pieces of about 50 additional parent asteroids (Scott 1979; Wasson 1990). This is a high number of parent bodies considering that the grouped irons, which compose nearly $88 \%$ of all irons, are believed to sample only 13 parent asteroids.

Within a given magmatic iron meteorite group, elemental variations are commonly attributed to the fractional crystallization history of the parent asteroidal core (e.g., Scott 1972). Models based on experimentally determined partitioning behaviors have had success at reproducing many of the observed elemental trends in magmatic iron meteorite groups by fractional crystallization (e.g., Jones and Drake 1983; Haack and Scott 1993; Chabot 2004). However, the experimental partitioning data on which these models are based are predominantly from systems with about $10 \mathrm{wt} \% \mathrm{Ni}$ (e.g., Jones and Drake 1983; Jones and Malvin 1990; Chabot et al. 2003). As shown in Fig. 1, though the large majority of iron meteorites contain $10 \mathrm{wt} \% \mathrm{Ni}$ or less, the $\mathrm{Ni}$ contents of iron meteorites also show considerable variation, ranging from 4 to $60 \mathrm{wt} \% \mathrm{Ni}$. It has not been demonstrated whether the crystallization models and the experimental data on which they are based are appropriate for understanding systems over such a large range of $\mathrm{Ni}$ contents. If $\mathrm{Ni}$ concentrations affect partitioning behavior, there may be the potential that, by 


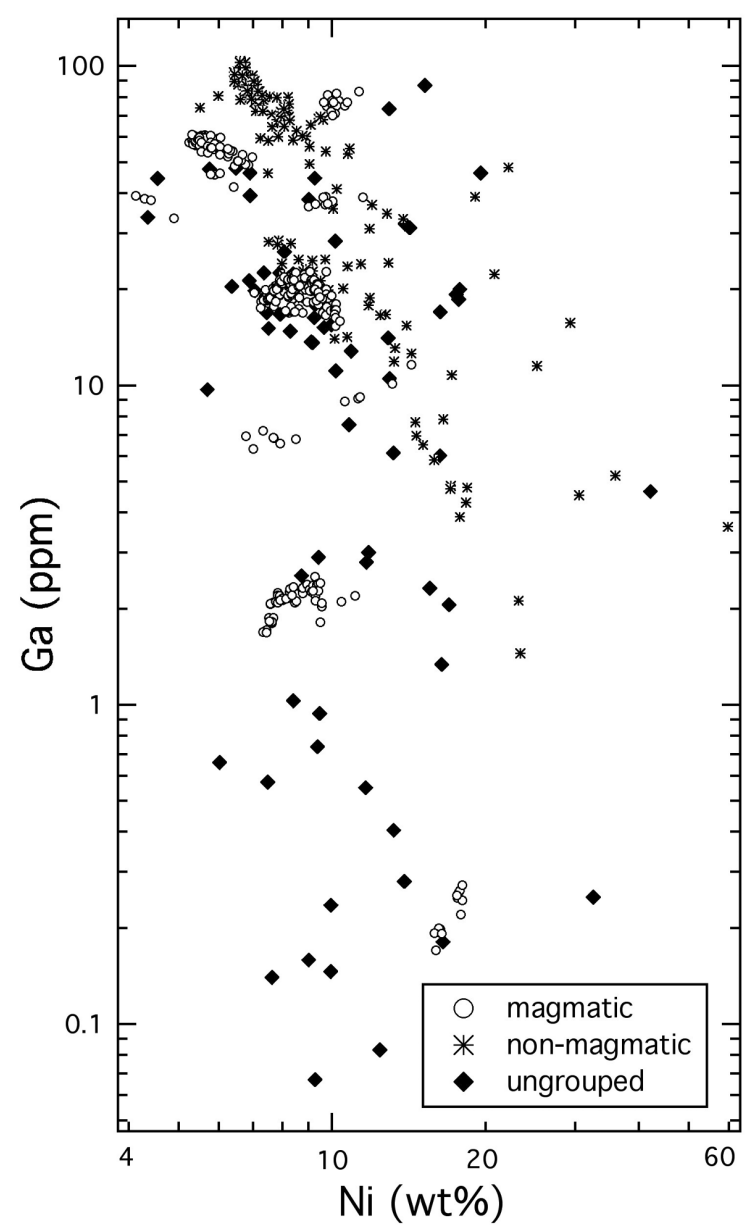

Fig. 1. Iron meteorites exhibit a large range in $\mathrm{Ni}$ concentrations, both for the magmatic and nonmagmatic groups as well as for ungrouped irons. The vast majority of iron meteorite compositional data are from an ongoing series of papers by J. T. Wasson and colleagues (Wasson 1967, 1969, 1970; Wasson and Kimberlin 1967; Wasson and Schaudy 1971; Schaudy et al. 1972; Scott et al. 1973; Scott and Wasson 1976; Kracher et al. 1980; Malvin et al. 1984; Wasson et al. 1989, 1998). Additional iron meteorite compositional studies are listed in the recent review of Haack and McCoy (2003).

including the effect, some of the higher Ni irons could be related to the main groups as previously unrecognized samples from the end of the fractional crystallization process.

With this motivation, we undertook an experimental study to investigate the effect of $\mathrm{Ni}$ on partitioning behavior, as applicable to the crystallization of iron meteorites.

\section{EXPERIMENTAL AND ANALYTICAL METHODS}

Experiments were conducted at the Applied Physics Laboratory in Maryland, USA, in a Deltech 1 atm vertical tube furnace using evacuated silica tubes, which is a similar experimental technique used by previous solid metal/liquid metal partitioning studies (e.g., Jones and Drake 1983; Chabot et al. 2003). Starting compositions were mixed from commercially purchased powders of $\mathrm{Fe}, \mathrm{Ni}$, and $\mathrm{FeS}$, with trace elements added as powders of $\mathrm{Ag}_{2} \mathrm{O}, \mathrm{As}, \mathrm{Au}, \mathrm{Bi}, \mathrm{Co}$, $\mathrm{Cu}, \mathrm{Ga}, \mathrm{Ge}, \mathrm{Ir}, \mathrm{Mo}, \mathrm{Os}, \mathrm{PbO}, \mathrm{Pd}, \mathrm{Pt}, \mathrm{Re}, \mathrm{Rh}, \mathrm{Ru}, \mathrm{Sb}, \mathrm{Sn}$, and $\mathrm{H}_{2} \mathrm{WO}_{4}$ at levels of about 100-500 ppm each. The choice of trace element powders was based on materials available in the laboratory at the time the experiments were conducted; however, whether the trace elements were added as metals, oxides, or acids does not seem to affect the partitioning results when doped at the low concentrations used in these experiments. For example, our results for $\mathrm{W}$ are consistent with those of Liu and Fleet (2001), who used high purity W metal powder as the added trace element.

In a departure from previous experimental studies, the starting mixtures were not contained in an alumina crucible, but rather were placed directly into a high-purity silica glass tube. The final metallic run products and the used silica tubes did not show any evidence of reactions between the silica glass and the starting powders. Additionally, as discussed in the next section, we have good agreement between our new experimental results using this method and the results from previous studies that have utilized alumina crucibles to contain the powders. Thus, the elimination of an alumina crucible from the experimental design seems to have no consequences on the partitioning results for this system.

After about $200 \mathrm{mg}$ of the starting mixture was placed in the tube, the tube was evacuated. During the evacuation process, the starting material was heated with a torch to drive off any excess volatiles. The tube was then sealed and lowered into the furnace. For runs below $1400^{\circ} \mathrm{C}$, the furnace temperature was first held near $1400{ }^{\circ} \mathrm{C}$ for about $2 \mathrm{~h}$, which enabled the starting mixture to become completely molten; the temperature was then lowered to the desired temperature for the experiment. For experiments at $1400{ }^{\circ} \mathrm{C}$ or higher, experiments were brought straight to the run temperature. Run durations varied inversely with the temperature, ranging from 1 to 8 days, and were based on similar previous experimental studies in which equilibrium was obtained (e.g., Jones and Drake 1983; Malvin et al. 1986; Chabot et al. 2003). Specifically, Malvin et al. (1986) observed similar partitioning values at $1250{ }^{\circ} \mathrm{C}$ for run durations varying from just 5 hours to 21 days. At the end of each experiment, the tube was removed from the furnace and immersed briefly in water, resulting in a quick quench of the run product. The run product, which commonly resembled a small metallic sphere, was mounted in epoxy, cut with a diamond saw, and polished for inspection and analysis.

Three sets of experiments were conducted. Experiments from the first set, detailed in Table 1, were run at a temperature of $1200^{\circ} \mathrm{C}$ and contained varying amounts of $\mathrm{Ni}$ in the starting powders. Initial Ni contents ranged from a Nifree run to a run with equal parts $\mathrm{Fe}$ and $\mathrm{Ni}$. The motivation was to hold all other variables constant while varying the $\mathrm{Ni}$ content in order to determine the effect of $\mathrm{Ni}$ on partitioning behavior. However, due to the phase relations, the amount of 
Table 1. Composition and partitioning data for experiments with variable Ni contents at $1200{ }^{\circ} \mathrm{C}$.

\begin{tabular}{|c|c|c|c|c|c|c|}
\hline Run no. & SS1 & SS2 & SS3 & SS4 & SS5 & SS6 \\
\hline $\mathrm{T}\left({ }^{\circ} \mathrm{C}\right)$ & 1198 & 1197 & 1194 & 1201 & 1197 & 1197 \\
\hline Duration (h) & 142 & 143 & 143 & 96 & 118 & 121 \\
\hline \multicolumn{7}{|l|}{ Liquid metal } \\
\hline $\mathrm{Fe}(\mathrm{wt} \%)$ & $67.1 \pm 1.7$ & $62.5 \pm 1.4$ & $58.2 \pm 1.7$ & $50.2 \pm 1.8$ & $46.0 \pm 2.4$ & $40.2 \pm 1.4$ \\
\hline $\mathrm{Ni}(\mathrm{wt} \%)$ & & $6.6 \pm 1.0$ & $14.4 \pm 1.6$ & $21.1 \pm 2.0$ & $31.6 \pm 1.6$ & $37.1 \pm 1.9$ \\
\hline $\mathrm{S}(\mathrm{wt} \%)$ & $30.1 \pm 1.7$ & $27.7 \pm 2.0$ & $25.2 \pm 2.1$ & $26.2 \pm 2.0$ & $20.1 \pm 2.4$ & $21.5 \pm 2.4$ \\
\hline $\mathrm{Ag}(\mathrm{ppm})$ & & & & $230 \pm 24$ & $230 \pm 30$ & \\
\hline As (ppm) & $36 \pm 2.2$ & $92 \pm 1.8$ & $74 \pm 1.2$ & $117 \pm 2.2$ & $185 \pm 6$ & $122 \pm 2$ \\
\hline $\mathrm{Au}(\mathrm{ppm})$ & $32 \pm 2$ & $73 \pm 0.6$ & $51 \pm 2.4$ & $70 \pm 4$ & $107 \pm 6$ & $75 \pm 4$ \\
\hline Co (ppm) & $36 \pm 2$ & $137 \pm 4$ & $157 \pm 8$ & $226 \pm 10$ & $378 \pm 14$ & $396 \pm 2$ \\
\hline $\mathrm{Cu}(\mathrm{ppm})$ & $610 \pm 50$ & $913 \pm 16$ & $660 \pm 70$ & $570 \pm 50$ & $440 \pm 50$ & $414 \pm 18$ \\
\hline $\mathrm{Ga}(\mathrm{ppm})$ & $3.7 \pm 0.8$ & $9.1 \pm 0.2$ & $0.6 \pm 0.2$ & $13 \pm 1.5$ & $32 \pm 4$ & $18 \pm 1.0$ \\
\hline $\mathrm{Ge}(\mathrm{ppm})$ & $3.4 \pm 0.6$ & $8.6 \pm 0.4$ & $7.9 \pm 2.4$ & $18 \pm 2.2$ & $52 \pm 6$ & $30 \pm 0.8$ \\
\hline Ir (ppm) & $0.2 \pm 0.07$ & $0.55 \pm 0.02$ & $0.43 \pm 0.18$ & $1.5 \pm 0.4$ & $3.0 \pm 0.4$ & $3.0 \pm 0.4$ \\
\hline Mo (ppm) & $52 \pm 1.6$ & $129 \pm 1.4$ & $90 \pm 6$ & $132 \pm 8$ & $177 \pm 4$ & $128 \pm 4$ \\
\hline Os (ppm) & $0.11 \pm 0.03$ & $0.39 \pm 0.04$ & $0.19 \pm 0.08$ & $0.80 \pm 0.15$ & $1.39 \pm 0.14$ & $1.4 \pm 0.2$ \\
\hline $\mathrm{Pb}(\mathrm{ppm})$ & & & & $470 \pm 50$ & & \\
\hline $\mathrm{Pd}(\mathrm{ppm})$ & $46 \pm 4$ & $112 \pm 3$ & $58 \pm 7$ & $86 \pm 4$ & $104 \pm 8$ & $67 \pm 2$ \\
\hline $\mathrm{Pt}(\mathrm{ppm})$ & $0.83 \pm 0.24$ & $2.2 \pm 0.2$ & $1.4 \pm 0.6$ & $4 \pm 0.6$ & $10 \pm 1.0$ & $8.5 \pm 0.8$ \\
\hline $\mathrm{Rh}(\mathrm{ppm})$ & & $0.040 \pm 0.003$ & $0.03 \pm 0.01$ & $0.06 \pm 0.01$ & $0.130 \pm 0.012$ & $0.13 \pm 0.01$ \\
\hline $\mathrm{Ru}(\mathrm{ppm})$ & $1.0 \pm 0.3$ & $3.2 \pm 0.2$ & $2.4 \pm 1.0$ & $7 \pm 1.2$ & $19 \pm 2$ & $14 \pm 0.8$ \\
\hline $\operatorname{Re}(\mathrm{ppm})$ & $0.14 \pm 0.04$ & $0.46 \pm 0.01$ & $0.3 \pm 0.1$ & $0.96 \pm 0.16$ & $2 \pm 0.2$ & $2.4 \pm 0.2$ \\
\hline $\mathrm{Sb}(\mathrm{ppm})$ & $137 \pm 16$ & $233 \pm 6$ & $165 \pm 22$ & $255 \pm 16$ & $319 \pm 26$ & $188 \pm 10$ \\
\hline $\mathrm{Sn}(\mathrm{ppm})$ & $125 \pm 16$ & $277 \pm 3$ & & $247 \pm 14$ & $304 \pm 20$ & $79 \pm 6$ \\
\hline $\mathrm{W}(\mathrm{ppm})$ & & $2.56 \pm 0.12$ & $1.8 \pm 0.6$ & $4 \pm 1$ & $7 \pm 1$ & $5.3 \pm 0.4$ \\
\hline \multicolumn{7}{|l|}{ Solid metal } \\
\hline $\mathrm{Fe}(\mathrm{wt} \%)$ & $98.9 \pm 0.23$ & $87 \pm 0.1$ & $76.9 \pm 0.14$ & $66.5 \pm 0.1$ & $55.8 \pm 0.22$ & $49.1 \pm 0.13$ \\
\hline $\mathrm{Ni}(\mathrm{wt} \%)$ & & $10.9 \pm 0.03$ & $21.5 \pm 0.03$ & $32.2 \pm 0.04$ & $42.0 \pm 0.01$ & $49.9 \pm 0.1$ \\
\hline $\mathrm{Ag}(\mathrm{ppm})$ & & & & $2.2 \pm 0.28$ & $6 \pm 4$ & \\
\hline As (ppm) & $113 \pm 2$ & $240 \pm 6$ & $137 \pm 9$ & $178 \pm 6$ & $119 \pm 4$ & $73 \pm 2$ \\
\hline $\mathrm{Au}(\mathrm{ppm})$ & $85 \pm 4$ & $222 \pm 6$ & $167 \pm 4$ & $205 \pm 2$ & $131 \pm 2$ & $109 \pm 4$ \\
\hline Co (ppm) & $169 \pm 2$ & $465 \pm 5$ & $556 \pm 20$ & $729 \pm 0.1$ & $788 \pm 4$ & $869 \pm 18$ \\
\hline $\mathrm{Cu}(\mathrm{ppm})$ & $88 \pm 2$ & $150 \pm 1.4$ & $153 \pm 2$ & $179 \pm 2$ & $171 \pm 2$ & $157 \pm 4$ \\
\hline $\mathrm{Ga}(\mathrm{ppm})$ & $84 \pm 1$ & $207 \pm 5$ & $22 \pm 1.4$ & $211 \pm 2$ & $179 \pm 10$ & $125 \pm 4$ \\
\hline $\mathrm{Ge}(\mathrm{ppm})$ & $139 \pm 8$ & $353 \pm 6$ & $262 \pm 22$ & $371 \pm 6$ & $307 \pm 12$ & $209 \pm 4$ \\
\hline Ir (ppm) & $110 \pm 30$ & $410 \pm 30$ & $377 \pm 8$ & $360 \pm 60$ & $377 \pm 8$ & $348 \pm 6$ \\
\hline Mo (ppm) & $207 \pm 10$ & $374 \pm 8$ & $290 \pm 15$ & $360 \pm 6$ & $316 \pm 22$ & $224 \pm 4$ \\
\hline Os (ppm) & $90 \pm 30$ & $400 \pm 50$ & $285 \pm 8$ & $290 \pm 70$ & $277 \pm 6$ & $264 \pm 4$ \\
\hline $\mathrm{Pb}(\mathrm{ppm})$ & & & & $0.4 \pm 0.08$ & & \\
\hline $\operatorname{Pd}(\mathrm{ppm})$ & $67 \pm 4$ & $172 \pm 5$ & $137 \pm 14$ & $161 \pm 1.4$ & $128 \pm 6$ & $94 \pm 2$ \\
\hline $\mathrm{Pt}(\mathrm{ppm})$ & $118 \pm 18$ & $339 \pm 11$ & $258 \pm 6$ & $330 \pm 20$ & $281 \pm 10$ & $265 \pm 4$ \\
\hline $\operatorname{Re}(\mathrm{ppm})$ & $93 \pm 8$ & $380 \pm 25$ & $307 \pm 9$ & $280 \pm 60$ & $302 \pm 4$ & $311 \pm 8$ \\
\hline $\mathrm{Rh}(\mathrm{ppm})$ & & $0.26 \pm 0.04$ & $0.45 \pm 0.04$ & $0.80 \pm 0.04$ & $1.0 \pm 0.05$ & $1.2 \pm 0.1$ \\
\hline $\mathrm{Ru}(\mathrm{ppm})$ & $130 \pm 10$ & $361 \pm 10$ & $269 \pm 4$ & $391 \pm 16$ & $390 \pm 22$ & $274 \pm 12$ \\
\hline $\mathrm{Sb}(\mathrm{ppm})$ & $35 \pm 2$ & $59 \pm 2.8$ & $46 \pm 2.8$ & $63 \pm 1$ & $60 \pm 2$ & $27 \pm 0.8$ \\
\hline Sn (ppm) & $41 \pm 0.2$ & $118 \pm 3$ & & $110 \pm 1$ & $97 \pm 2$ & $21 \pm 2$ \\
\hline $\mathrm{W}(\mathrm{ppm})$ & & $223 \pm 7$ & $185 \pm 6$ & $226 \pm 15$ & $167 \pm 2$ & $161 \pm 2$ \\
\hline \multicolumn{7}{|c|}{ Partition coefficients } \\
\hline $\mathrm{D}(\mathrm{Ni})$ & & $1.6 \pm 0.11$ & $1.5 \pm 0.08$ & $1.5 \pm 0.07$ & $1.3 \pm 0.03$ & $1.3 \pm 0.04$ \\
\hline $\mathrm{D}(\mathrm{Ag})$ & & & & $0.01 \pm 0.002$ & $0.03 \pm 0.016$ & \\
\hline $\mathrm{D}(\mathrm{As})$ & $3.2 \pm 0.2$ & $2.6 \pm 0.1$ & $1.9 \pm 0.12$ & $1.52 \pm 0.06$ & $0.64 \pm 0.02$ & $0.60 \pm 0.04$ \\
\hline $\mathrm{D}(\mathrm{Au})$ & $2.67 \pm 0.19$ & $3.0 \pm 0.1$ & $3.25 \pm 0.22$ & $2.9 \pm 0.16$ & $1.2 \pm 0.1$ & $1.5 \pm 0.1$ \\
\hline $\mathrm{D}(\mathrm{Co})$ & $4.63 \pm 0.26$ & $3.4 \pm 0.1$ & $3.55 \pm 0.24$ & $3.22 \pm 0.15$ & $2.1 \pm 0.1$ & $2.19 \pm 0.02$ \\
\hline $\mathrm{D}(\mathrm{Cu})$ & $0.14 \pm 0.014$ & $0.165 \pm 0.004$ & $0.230 \pm 0.026$ & $0.31 \pm 0.03$ & $0.39 \pm 0.04$ & $0.38 \pm 0.05$ \\
\hline $\mathrm{D}(\mathrm{Ga})$ & $23 \pm 4$ & $23 \pm 1$ & $35 \pm 13$ & $16 \pm 1.8$ & $5.5 \pm 0.8$ & $6.9 \pm 0.06$ \\
\hline
\end{tabular}


Table 1. Continued. Composition and partitioning data for experiments with variable Ni contents at $1200{ }^{\circ} \mathrm{C}$.

\begin{tabular}{lcccccc}
\hline Run no. & SS1 & SS2 & SS3 & SS4 & SS5 & SS6 \\
\hline $\mathrm{D}(\mathrm{Ge})$ & $41 \pm 8$ & $41 \pm 1.6$ & $33 \pm 10.4$ & $20 \pm 2.6$ & $6.0 \pm 0.8$ & $7.0 \pm 0.03$ \\
$\mathrm{D}(\mathrm{Ir})$ & $580 \pm 240$ & $760 \pm 60$ & $870 \pm 370$ & $240 \pm 60$ & $126 \pm 18$ & $115 \pm 0.12$ \\
$\mathrm{D}(\mathrm{Mo})$ & $3.9 \pm 0.2$ & $2.9 \pm 0.08$ & $3.2 \pm 0.28$ & $2.72 \pm 0.17$ & $1.78 \pm 0.14$ & $1.75 \pm 0.04$ \\
$\mathrm{D}(\mathrm{Os})$ & $890 \pm 390$ & $1030 \pm 160$ & $1490 \pm 640$ & $360 \pm 120$ & $200 \pm 20$ & $189 \pm 0.14$ \\
$\mathrm{D}(\mathrm{Pb})$ & & & $0.0009 \pm 0.0002$ & & \\
$\mathrm{D}(\mathrm{Pd})$ & $1.47 \pm 0.14$ & $1.53 \pm 0.06$ & $2.4 \pm 0.4$ & $1.89 \pm 0.08$ & $1.24 \pm 0.11$ & $1.41 \pm 0.04$ \\
$\mathrm{D}(\mathrm{Pt})$ & $140 \pm 50$ & $154 \pm 8$ & $190 \pm 80$ & $83 \pm 14$ & $27 \pm 3.0$ & $31 \pm 0.1$ \\
$\mathrm{D}(\mathrm{Re})$ & $690 \pm 230$ & $840 \pm 50$ & $1150 \pm 420$ & $290 \pm 80$ & $135 \pm 16$ & $130 \pm 0.1$ \\
$\mathrm{D}(\mathrm{Rh})$ & & $6.5 \pm 1.0$ & $13.1 \pm 2.6$ & $14 \pm 1.8$ & $8 \pm 1$ & $9.4 \pm 0.1$ \\
$\mathrm{D}(\mathrm{Ru})$ & $130 \pm 40$ & $113 \pm 6$ & $110 \pm 50$ & $57 \pm 10$ & $20 \pm 3$ & $19.7 \pm 0.1$ \\
$\mathrm{D}(\mathrm{Sb})$ & $0.26 \pm 0.04$ & $0.254 \pm 0.014$ & $0.279 \pm 0.04$ & $0.25 \pm 0.016$ & $0.19 \pm 0.018$ & $0.14 \pm 0.06$ \\
$\mathrm{D}(\mathrm{Sn})$ & $0.33 \pm 0.04$ & $0.424 \pm 0.012$ & & $0.45 \pm 0.026$ & $0.32 \pm 0.020$ & $0.26 \pm 0.16$ \\
$\mathrm{D}(\mathrm{W})$ & & $87 \pm 4$ & $100 \pm 40$ & $58 \pm 10$ & $24 \pm 2.4$ & $30 \pm 0.1$ \\
\hline $\mathrm{D}$ & & & & & \\
\hline
\end{tabular}

Data for $\mathrm{Fe}, \mathrm{Ni}$, and $\mathrm{S}$ are from electron microprobe analysis. All other data are from LA ICP-MS.

Errors are $\pm 2 \sigma$ of the mean.

Table 2. Composition and partitioning for experiments in the Ni-S system.

\begin{tabular}{|c|c|c|c|c|c|c|}
\hline Run no. & NN4 & NN5 & NN6 & NN7 & NN8 & NN9 \\
\hline $\mathrm{T}\left({ }^{\circ} \mathrm{C}\right)$ & 950 & 1050 & 1150 & 1227 & 1327 & 1400 \\
\hline Duration (h) & 192 & 191 & 145 & 95 & 92 & 25 \\
\hline \multicolumn{7}{|l|}{ Liquid metal } \\
\hline $\mathrm{Ni}(\mathrm{wt} \%)$ & $80.1 \pm 1.9$ & $81.1 \pm 1.7$ & $82.4 \pm 1.6$ & $84.2 \pm 2.0$ & $92.5 \pm 1.6$ & $97 \pm 1$ \\
\hline $\mathrm{S}(\mathrm{wt} \%)$ & $19.5 \pm 1.8$ & $18.0 \pm 1.6$ & $16.5 \pm 1.5$ & $15.0 \pm 1.9$ & $7.4 \pm 1.6$ & $3 \pm 1$ \\
\hline $\mathrm{Ag}(\mathrm{ppm})$ & $236 \pm 24$ & $114 \pm 14$ & $115 \pm 16$ & $313 \pm 18$ & $110 \pm 14$ & $400 \pm 80$ \\
\hline As (ppm) & $168 \pm 8$ & $114 \pm 4$ & $141 \pm 14$ & $350 \pm 12$ & $189 \pm 14$ & $350 \pm 30$ \\
\hline $\mathrm{Au}(\mathrm{ppm})$ & $203 \pm 16$ & $128 \pm 16$ & $178 \pm 22$ & $401 \pm 18$ & $221 \pm 18$ & $340 \pm 40$ \\
\hline $\mathrm{Bi}(\mathrm{ppm})$ & & $214 \pm 22$ & $240 \pm 30$ & $870 \pm 50$ & $410 \pm 50$ & $640 \pm 100$ \\
\hline Co (ppm) & $342 \pm 10$ & $391 \pm 18$ & $405 \pm 28$ & $523 \pm 18$ & $750 \pm 30$ & $1020 \pm 30$ \\
\hline $\mathrm{Cu}(\mathrm{ppm})$ & $222 \pm 14$ & $127 \pm 6$ & $127 \pm 12$ & $287 \pm 10$ & $171 \pm 10$ & $396 \pm 16$ \\
\hline Ga (ppm) & & $0.22 \pm 0.02$ & $0.89 \pm 0.16$ & $4.1 \pm 0.2$ & $53 \pm 1.8$ & $108 \pm 2$ \\
\hline Ge (ppm) & $18 \pm 3$ & $26 \pm 4$ & $39 \pm 6$ & $75 \pm 4$ & $98 \pm 2$ & $198 \pm 4$ \\
\hline Ir (ppm) & $1.0 \pm 0.3$ & $2.0 \pm 0.4$ & $7.3 \pm 2.2$ & $11 \pm 1$ & $37 \pm 4$ & $88 \pm 6$ \\
\hline Mo (ppm) & & $1.0 \pm 0.1$ & $2.6 \pm 0.12$ & $5.0 \pm 0.4$ & $55 \pm 2$ & $159 \pm 2$ \\
\hline Os (ppm) & $0.3 \pm 0.06$ & $0.6 \pm 0.1$ & $3.1 \pm 0.9$ & $4.3 \pm 0.5$ & $15 \pm 2$ & $51 \pm 4$ \\
\hline $\mathrm{Pb}(\mathrm{ppm})$ & & & $300 \pm 40$ & $920 \pm 60$ & $480 \pm 60$ & $750 \pm 120$ \\
\hline $\mathrm{Pd}(\mathrm{ppm})$ & $145 \pm 4$ & $116 \pm 2$ & $144 \pm 4$ & $271 \pm 4$ & $167 \pm 6$ & $274 \pm 20$ \\
\hline $\mathrm{Pt}(\mathrm{ppm})$ & $7.6 \pm 2.4$ & $8.0 \pm 1.0$ & $22 \pm 4$ & $29 \pm 1.0$ & $59 \pm 4$ & $111 \pm 2$ \\
\hline $\operatorname{Re}(\mathrm{ppm})$ & $0.37 \pm 0.12$ & $0.64 \pm 0.04$ & $3 \pm 1$ & $5.1 \pm 0.2$ & $22 \pm 2$ & $74 \pm 6$ \\
\hline $\mathrm{Rh}$ (ppm) & $0.20 \pm 0.02$ & $0.24 \pm 0.04$ & $0.28 \pm 0.04$ & $0.39 \pm 0.02$ & $0.73 \pm 0.04$ & $0.94 \pm 0.04$ \\
\hline $\mathrm{Ru}(\mathrm{ppm})$ & & $25 \pm 5$ & $22 \pm 4$ & $35 \pm 8$ & $41 \pm 2$ & $64 \pm 6$ \\
\hline $\mathrm{Sb}(\mathrm{ppm})$ & $230 \pm 14$ & $165 \pm 14$ & $240 \pm 30$ & $630 \pm 40$ & $330 \pm 30$ & $500 \pm 10$ \\
\hline $\mathrm{Sn}(\mathrm{ppm})$ & $221 \pm 16$ & $115 \pm 10$ & $202 \pm 24$ & $630 \pm 40$ & $410 \pm 50$ & $590 \pm 80$ \\
\hline W (ppm) & & & & & $1.6 \pm 0.2$ & $17 \pm 1.0$ \\
\hline \multicolumn{7}{|l|}{ Solid metal } \\
\hline $\mathrm{Ni}(\mathrm{wt} \%)$ & $99.7 \pm 0.5$ & $99.5 \pm 0.1$ & $98.9 \pm 0.3$ & $99.7 \pm 0.6$ & $99.4 \pm 0.1$ & $99.8 \pm 0.4$ \\
\hline $\mathrm{Ag}(\mathrm{ppm})$ & $1.5 \pm 0.3$ & $1.7 \pm 0.18$ & $2.1 \pm 0.2$ & $7.0 \pm 0.4$ & $5.1 \pm 0.1$ & $21 \pm 2$ \\
\hline As (ppm) & $171 \pm 16$ & $90 \pm 2$ & $66 \pm 2$ & $106 \pm 6$ & $44 \pm 2$ & $70 \pm 24$ \\
\hline $\mathrm{Au}(\mathrm{ppm})$ & $144 \pm 10$ & $89 \pm 2$ & $79 \pm 0.15$ & $140 \pm 2$ & $67 \pm 2$ & $89 \pm 20$ \\
\hline Bi (ppm) & & $0.15 \pm 0.04$ & $0.22 \pm 0.002$ & $1.0 \pm 0.1$ & $0.9 \pm 0.1$ & $2.7 \pm 0.1$ \\
\hline Co (ppm) & $1230 \pm 50$ & $1151 \pm 12$ & $1157 \pm 12$ & $1290 \pm 15$ & $1244 \pm 20$ & $1233 \pm 24$ \\
\hline $\mathrm{Cu}(\mathrm{ppm})$ & $175 \pm 12$ & $115 \pm 2$ & $107 \pm 2$ & $221 \pm 7$ & $129 \pm 2$ & $286 \pm 18$ \\
\hline $\mathrm{Ga}$ (ppm) & & $20 \pm 2$ & $8.0 \pm 1.0$ & $18 \pm 5$ & $79 \pm 1$ & $101 \pm 8$ \\
\hline Ge (ppm) & $470 \pm 60$ & $290 \pm 10$ & $248 \pm 2$ & $250 \pm 15$ & $120 \pm 8$ & $153 \pm 24$ \\
\hline Ir (ppm) & $1060 \pm 170$ & $470 \pm 10$ & $632 \pm 9$ & $345 \pm 18$ & $251 \pm 4$ & $235 \pm 28$ \\
\hline Mo (ppm) & & $10 \pm 1$ & $18 \pm 2.8$ & $22 \pm 2.4$ & $143 \pm 6$ & $207 \pm 4$ \\
\hline
\end{tabular}


Table 2. Continued. Composition and partitioning for experiments in the Ni-S system.

\begin{tabular}{|c|c|c|c|c|c|c|}
\hline Run no. & NN4 & NN5 & NN6 & NN7 & NN8 & NN9 \\
\hline Os (ppm) & $780 \pm 240$ & $299 \pm 12$ & $478 \pm 20$ & $253 \pm 10$ & $166 \pm 8$ & $172 \pm 28$ \\
\hline $\mathrm{Pb}(\mathrm{ppm})$ & & & $0.5 \pm 0.1$ & $2.4 \pm 0.14$ & $2.54 \pm 0.12$ & $6.4 \pm 0.8$ \\
\hline $\mathrm{Pd}(\mathrm{ppm})$ & $100 \pm 2$ & $77 \pm 2$ & $83 \pm 2$ & $127 \pm 1.4$ & $74 \pm 4$ & $106 \pm 14$ \\
\hline $\mathrm{Pt}(\mathrm{ppm})$ & $900 \pm 40$ & $388 \pm 8$ & $451 \pm 5$ & $287 \pm 6$ & $168 \pm 2$ & $167 \pm 4$ \\
\hline $\operatorname{Re}(p p m)$ & $1030 \pm 280$ & $47 \pm 30$ & $593 \pm 12$ & $344 \pm 14$ & $252 \pm 8$ & $270 \pm 50$ \\
\hline $\mathrm{Rh}$ (ppm) & $1.4 \pm 0.1$ & $1.2 \pm 0.1$ & $1.2 \pm 0.1$ & $1.2 \pm 0.2$ & $1.1 \pm 0.2$ & $1.1 \pm 0.1$ \\
\hline $\mathrm{Ru}(\mathrm{ppm})$ & $700 \pm 30$ & $351 \pm 4$ & $409 \pm 6$ & $309 \pm 6$ & $195 \pm 2$ & $199 \pm 8$ \\
\hline $\mathrm{Sb}(\mathrm{ppm})$ & $67 \pm 6$ & $45 \pm 2$ & $39 \pm 1$ & $75 \pm 2.8$ & $35 \pm 1$ & $52 \pm 16$ \\
\hline $\mathrm{Sn}(\mathrm{ppm})$ & $69 \pm 4$ & $37 \pm 2$ & $39 \pm 2$ & $85 \pm 1.2$ & $48 \pm 2$ & $65 \pm 22$ \\
\hline W (ppm) & & & & & $11 \pm 1$ & $39 \pm 4$ \\
\hline \multicolumn{7}{|c|}{ Partition coefficients } \\
\hline $\mathrm{D}(\mathrm{Ni})$ & $1.2 \pm 0.01$ & $1.2 \pm 0.01$ & $1.2 \pm 0.01$ & $1.2 \pm 0.01$ & $1.1 \pm 0.01$ & $1.0 \pm 0.01$ \\
\hline $\mathrm{D}(\mathrm{Ag})$ & $0.006 \pm 0.0012$ & $0.015 \pm 0.002$ & $0.02 \pm 0.002$ & $0.02 \pm 0.002$ & $0.046 \pm 0.001$ & $0.05 \pm 0.02$ \\
\hline $\mathrm{D}(\mathrm{As})$ & $1.0 \pm 0.1$ & $0.79 \pm 0.04$ & $0.47 \pm 0.04$ & $0.30 \pm 0.02$ & $0.23 \pm 0.02$ & $0.2 \pm 0.1$ \\
\hline $\mathrm{D}(\mathrm{Au})$ & $0.7 \pm 0.1$ & $0.7 \pm 0.1$ & $0.4 \pm 0.1$ & $0.35 \pm 0.016$ & $0.30 \pm 0.02$ & $0.3 \pm 0.1$ \\
\hline $\mathrm{D}(\mathrm{Bi})$ & & $0.0007 \pm 0.0002$ & $0.0009 \pm 0.0002$ & $0.0011 \pm 0.0002$ & $0.0021 \pm 0.0004$ & $0.004 \pm 0.001$ \\
\hline $\mathrm{D}(\mathrm{Co})$ & $3.60 \pm 0.20$ & $2.95 \pm 0.14$ & $2.86 \pm 0.20$ & $2.47 \pm 0.10$ & $1.7 \pm 0.1$ & $1.20 \pm 0.04$ \\
\hline $\mathrm{D}(\mathrm{Cu})$ & $0.78 \pm 0.07$ & $0.90 \pm 0.04$ & $0.84 \pm 0.07$ & $0.77 \pm 0.04$ & $0.75 \pm 0.04$ & $0.7 \pm 0.1$ \\
\hline $\mathrm{D}(\mathrm{Ga})$ & & $93 \pm 16$ & $8.4 \pm 1.8$ & $4.4 \pm 1.3$ & $1.5 \pm 0.1$ & $0.9 \pm 0.1$ \\
\hline $\mathrm{D}(\mathrm{Ge})$ & $26 \pm 6$ & $11.2 \pm 1.4$ & $6.4 \pm 1.0$ & $3.3 \pm 0.24$ & $1.2 \pm 0.1$ & $0.77 \pm 0.12$ \\
\hline $\mathrm{D}(\mathrm{Ir})$ & $1900 \pm 400$ & $260 \pm 40$ & $90 \pm 30$ & $31 \pm 2$ & $7 \pm 1$ & $2.7 \pm 0.4$ \\
\hline $\mathrm{D}(\mathrm{Mo})$ & & $10.4 \pm 1.2$ & $6.8 \pm 1.1$ & $4.8 \pm 0.6$ & $2.59 \pm 0.14$ & $1.3 \pm 0.04$ \\
\hline $\mathrm{D}(\mathrm{Os})$ & $2570 \pm 950$ & $550 \pm 80$ & $160 \pm 40$ & $59 \pm 8$ & $11 \pm 1.6$ & $3 \pm 1$ \\
\hline $\mathrm{D}(\mathrm{Pb})$ & & & $0.0017 \pm 0.0004$ & $0.0030 \pm 0.0002$ & $0.005 \pm 0.001$ & $0.01 \pm 0.002$ \\
\hline $\mathrm{D}(\mathrm{Pd})$ & $0.69 \pm 0.022$ & $0.662 \pm 0.016$ & $0.57 \pm 0.016$ & $0.47 \pm 0.01$ & $0.45 \pm 0.03$ & $0.4 \pm 0.1$ \\
\hline $\mathrm{D}(\mathrm{Pt})$ & $120 \pm 40$ & $48 \pm 6$ & $21 \pm 5$ & $9.9 \pm 0.4$ & $2.84 \pm 0.16$ & $1.51 \pm 0.04$ \\
\hline $\mathrm{D}(\mathrm{Re})$ & $2820 \pm 1140$ & $720 \pm 110$ & $220 \pm 60$ & $67 \pm 4$ & $11.5 \pm 1.2$ & $4 \pm 1$ \\
\hline $\mathrm{D}(\mathrm{Rh})$ & $7 \pm 1$ & $5.1 \pm 0.7$ & $5 \pm 1$ & $3.1 \pm 0.4$ & $1.57 \pm 0.18$ & $1.14 \pm 0.12$ \\
\hline $\mathrm{D}(\mathrm{Ru})$ & $28 \pm 6$ & $15.9 \pm 2.6$ & $11.8 \pm 2.6$ & $8 \pm 1$ & $3.03 \pm 0.28$ & $1.56 \pm 0.10$ \\
\hline $\mathrm{D}(\mathrm{Sb})$ & $0.29 \pm 0.03$ & $0.273 \pm 0.026$ & $0.17 \pm 0.02$ & $0.12 \pm 0.01$ & $0.108 \pm 0.012$ & $0.10 \pm 0.04$ \\
\hline $\mathrm{D}(\mathrm{Sn})$ & $0.31 \pm 0.03$ & $0.32 \pm 0.04$ & $0.19 \pm 0.024$ & $0.13 \pm 0.01$ & $0.117 \pm 0.014$ & $0.11 \pm 0.04$ \\
\hline $\mathrm{D}(\mathrm{W})$ & & & & & $7 \pm 1$ & $2.30 \pm 0.26$ \\
\hline
\end{tabular}

Data for Ni and S are from electron microprobe analysis. All other data are from LA ICP-MS.

Errors are $\pm 2 \sigma$ of the mean.

Ni also influenced the $\mathrm{S}$ content of the equilibrium metallic liquid in each experiment, which is consistent with the Fe-Ni$\mathrm{S}$ phase diagram (Hsieh et al. 1982). Consequently, these experiments resulted in run products with both varying $\mathrm{Ni}$ contents and metallic liquid $\mathrm{S}$ contents, which, though relevant to many iron meteorite compositions, created a major complication for isolating the effect of $\mathrm{Ni}$. Sulfur is believed to have been a major component of many asteroidal cores (e.g., Chabot 2004), and the presence of S strongly influences the speciation in the metallic liquid, which is an important influence on partitioning behavior (Jones and Malvin 1990; Chabot and Jones 2003). Thus, including $S$ in the experiments may have complicated the interpretation of the effect of $\mathrm{Ni}$, but it was a necessary complication to investigate a system relevant to the crystallization of iron meteorites.

The second set of experiments examined the extreme end-member composition of the Fe-free Ni-S system; details about these runs are provided in Table 2. Though an Fe-free system is not directly applicable to iron meteorite compositions, the rationale was that by exploring the simplified $\mathrm{Ni}-\mathrm{S}$ system, any effect from $\mathrm{Ni}$ would be enhanced when compared to previous studies conducted in the low-Ni, predominantly $\mathrm{Fe}-\mathrm{S}$ system. If an element were observed to partition similarly in the Fe-S and Ni-S systems, then intermediate compositions, having a range of $\mathrm{Fe}$ to $\mathrm{Ni}$ ratios, would be expected to also partition similarly. To produce a range of $\mathrm{S}$ contents in the equilibrium metallic liquid, the run temperature of these experiments ranged from 950 to $1400{ }^{\circ} \mathrm{C}$.

As discussed in the next section, partitioning behaviors in the Ni-S system were observed to be significantly different than in the low-Ni Fe-S system for some trace elements of interest to iron meteorites. Thus, a third set of experiments was conducted that contained about equal parts of $\mathrm{Fe}$ and $\mathrm{Ni}$. As shown in Fig. 1, a 1:1 Fe:Ni ratio by weight is nearly the most Ni-rich composition of any iron meteorite. It was expected that partitioning behavior in this 1:1 (Fe:Ni)-S system would fall between the extreme end-member 

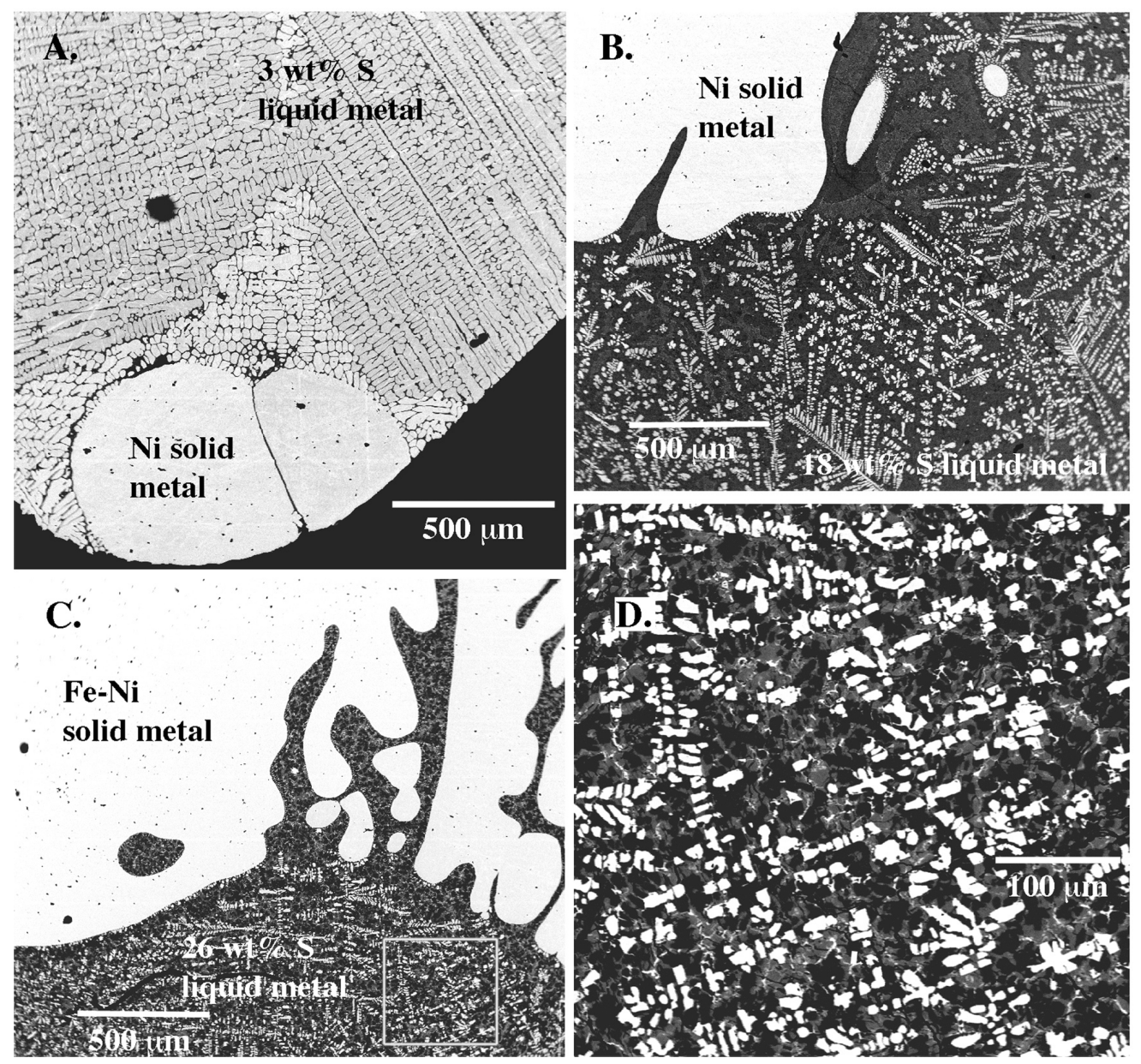

Fig. 2. Backscattered electron (BSE) images illustrate the range of experimental run products. a) Run \#NN9, conducted in the Ni-S system, shows the homogenous solid metal clearly distinguished from the metallic liquid with $3 \mathrm{wt} \% \mathrm{~S}$. Areas of different brightness in the quenched metallic liquid are due to crystal orientations during quenching; analytical measurements show that there are no compositional differences between these areas in the metallic liquid. b) The metallic liquid in run \#NN5, also conducted in the Ni-S system, contains more $\mathrm{S}$ (18 wt\%) than \#NN9, which results in the different metallic liquid quench textures seen in the two runs. c) Run \#SS4, conducted in the Fe-Ni-S system, has equilibrium solid metal and liquid metal. d) A close-up of the metallic liquid quench texture of \#SS4 (white box drawn in C) shows FeNi dendrites (brightest phase) surrounded by S-rich phases (both light and dark gray phases). The S-rich phases contain varying amount of Fe, $\mathrm{Ni}$, and $\mathrm{S}$, with up to about $36 \mathrm{wt} \% \mathrm{~S}$.

behaviors observed for the Ni-S and Fe-S systems. However, these experiments were conducted to determine if the effect of $\mathrm{Ni}$ was still significant when dealing with a composition relevant to iron meteorites, as compared to the end-member $\mathrm{Ni}-\mathrm{S}$ system. As given in Table 3, experiments were conducted at temperatures ranging from 1100 to $1350{ }^{\circ} \mathrm{C}$ to produce a range of metallic liquid $\mathrm{S}$ concentrations; in all experiments, the Fe:Ni ratio was held constant at 1:1.

Experimental charges were examined using an electron microprobe, either the Smithsonian Institution National Museum of Natural History JEOL JXA 8900R or the Carnegie Institution of Washington JEOL 8900L. Figure 2 shows backscattered electron images of some typical experimental run products. As seen in Fig. 2, solid metal and liquid metal formed well-separated, easily distinguished phases. The solid metal was homogenous while the liquid metal quenched to a multiphase assemblage with a dendritic texture characteristic of S-bearing metallic melts; the specific features of each quenched liquid depended on the $\mathrm{S}, \mathrm{Ni}$, and Fe contents of the bulk liquid, with diverse examples given in Fig. 2. The concentrations of the major elements of $\mathrm{Fe}, \mathrm{Ni}$, and $\mathrm{S}$ were determined by electron microprobe using beam conditions of $15-20 \mathrm{kV}$ and 20-30 nA with counting times of $30 \mathrm{~s}$. Either a defocused beam $20 \mu \mathrm{m}$ in diameter was used or the beam was rastered over a $20 \times 20 \mu \mathrm{m}$ area. The solid metal was homogenous; different analysis points yielded similar compositions and showed no evidence of gradients or zoning. Ten to 25 points were averaged to determine the composition of the solid metal in each experiment. The multiphased quench texture of the metallic liquid required that a larger number of analysis points be taken in order to reliably measure the bulk liquid composition; 30-50 points were 
Table 3. Composition and partitioning data for experiments with 1:1 Fe:Ni ratios.

\begin{tabular}{|c|c|c|c|c|}
\hline Run no. & DD2 & DD6 & DD3 & DD5 \\
\hline $\mathrm{T}\left({ }^{\circ} \mathrm{C}\right)$ & 1107 & 1278 & 1305 & 1355 \\
\hline Duration (h) & 120 & 137 & 51 & 24 \\
\hline \multicolumn{5}{|l|}{ Liquid metal } \\
\hline $\mathrm{Fe}(\mathrm{wt} \%)$ & $39.5 \pm 0.9$ & $43.4 \pm 0.8$ & $42.9 \pm 0.7$ & $45.9 \pm 0.5$ \\
\hline $\mathrm{Ni}(\mathrm{wt} \%)$ & $33.4 \pm 1.1$ & $37.5 \pm 1.0$ & $42.5 \pm 1.0$ & $46.3 \pm 0.7$ \\
\hline $\mathrm{S}(\mathrm{wt} \%)$ & $25.3 \pm 1.5$ & $19.0 \pm 1.3$ & $14.2 \pm 1.6$ & $7.1 \pm 1.1$ \\
\hline $\mathrm{Ag}(\mathrm{ppm})$ & $360 \pm 90$ & $100 \pm 40$ & $190 \pm 50$ & $120 \pm 50$ \\
\hline As (ppm) & $155 \pm 11$ & $83 \pm 15$ & $200 \pm 20$ & $75 \pm 13$ \\
\hline $\mathrm{Au}(\mathrm{ppm})$ & $125 \pm 30$ & $56 \pm 15$ & $200 \pm 18$ & $88 \pm 7$ \\
\hline $\mathrm{Bi}(\mathrm{ppm})$ & & $430 \pm 150$ & $500 \pm 130$ & $280 \pm 90$ \\
\hline $\mathrm{Co}(\mathrm{ppm})$ & $368 \pm 40$ & $280 \pm 20$ & $550 \pm 40$ & $430 \pm 40$ \\
\hline $\mathrm{Cu}(\mathrm{ppm})$ & $500 \pm 40$ & $220 \pm 50$ & $340 \pm 40$ & $190 \pm 30$ \\
\hline $\mathrm{Ga}(\mathrm{ppm})$ & & $14 \pm 4$ & $75 \pm 11$ & $31 \pm 4$ \\
\hline $\mathrm{Ge}$ (ppm) & & $26 \pm 7$ & $130 \pm 20$ & $65 \pm 8$ \\
\hline Ir (ppm) & & $2.6 \pm 0.8$ & $52 \pm 16$ & $25 \pm 8$ \\
\hline Mo (ppm) & $179 \pm 14$ & $72 \pm 7$ & $200 \pm 11$ & $100 \pm 14$ \\
\hline Os (ppm) & & $1.5 \pm 0.2$ & $27 \pm 9$ & $18 \pm 8$ \\
\hline $\mathrm{Pb}(\mathrm{ppm})$ & $580 \pm 110$ & $520 \pm 20$ & $530 \pm 140$ & $330 \pm 110$ \\
\hline $\mathrm{Pd}(\mathrm{ppm})$ & $110 \pm 30$ & $53 \pm 10$ & $154 \pm 4$ & $60 \pm 4$ \\
\hline $\mathrm{Pt}(\mathrm{ppm})$ & & $6.0 \pm 1.7$ & $67 \pm 17$ & $28 \pm 6$ \\
\hline $\operatorname{Re}(\mathrm{ppm})$ & & $2.0 \pm 0.4$ & $26 \pm 8$ & $18 \pm 7$ \\
\hline $\mathrm{Rh}(\mathrm{ppm})$ & & $0.13 \pm 0.05$ & $0.33 \pm 0.11$ & $0.34 \pm 0.09$ \\
\hline $\mathrm{Ru}(\mathrm{ppm})$ & & $9 \pm 3$ & $80 \pm 20$ & $38 \pm 10$ \\
\hline $\mathrm{Sb}(\mathrm{ppm})$ & $330 \pm 60$ & $300 \pm 60$ & $330 \pm 90$ & $180 \pm 60$ \\
\hline $\mathrm{Sn}(\mathrm{ppm})$ & $400 \pm 80$ & $200 \pm 30$ & $300 \pm 60$ & $180 \pm 50$ \\
\hline $\mathrm{W}(\mathrm{ppm})$ & & $4.3 \pm 0.9$ & $45 \pm 10$ & $17 \pm 5$ \\
\hline \multicolumn{5}{|l|}{ Solid metal } \\
\hline $\mathrm{Fe}(\mathrm{wt} \%)$ & $49.3 \pm 0.1$ & $52.5 \pm 0.3$ & $49.9 \pm 0.2$ & $50.0 \pm 0.1$ \\
\hline $\mathrm{Ni}(\mathrm{wt} \%)$ & $49.1 \pm 0.1$ & $46.7 \pm 0.2$ & $49.4 \pm 0.2$ & $49.2 \pm 0.1$ \\
\hline $\mathrm{Ag}(\mathrm{ppm})$ & $3.7 \pm 0.5$ & $2.4 \pm 0.2$ & $9 \pm 2$ & $6.7 \pm 1.3$ \\
\hline As (ppm) & $180 \pm 14$ & $40 \pm 2$ & $57 \pm 8$ & $19 \pm 4$ \\
\hline $\mathrm{Au}(\mathrm{ppm})$ & $320 \pm 12$ & $47 \pm 1$ & $120 \pm 20$ & $40 \pm 7$ \\
\hline Bi (ppm) & & $0.45 \pm 0.12$ & $0.7 \pm 0.1$ & $0.58 \pm 0.02$ \\
\hline Co (ppm) & $960 \pm 20$ & $539 \pm 8$ & $890 \pm 14$ & $580 \pm 40$ \\
\hline $\mathrm{Cu}(\mathrm{ppm})$ & $200 \pm 11$ & $103 \pm 6$ & $191 \pm 6$ & $108 \pm 9$ \\
\hline $\mathrm{Ga}(\mathrm{ppm})$ & & $43.3 \pm 0.6$ & $138 \pm 4$ & $37 \pm 3$ \\
\hline Ge (ppm) & & $77 \pm 7$ & $220 \pm 20$ & $69 \pm 7$ \\
\hline Ir (ppm) & & $65 \pm 5$ & $540 \pm 80$ & $130 \pm 20$ \\
\hline Mo (ppm) & $400 \pm 30$ & $107 \pm 10$ & $280 \pm 40$ & $106 \pm 8$ \\
\hline Os (ppm) & & $53 \pm 7$ & $360 \pm 40$ & $130 \pm 20$ \\
\hline $\mathrm{Pb}(\mathrm{ppm})$ & $0.51 \pm 0.14$ & $1.2 \pm 0.2$ & $2.0 \pm 0.3$ & $2.0 \pm 0.5$ \\
\hline $\mathrm{Pd}(\mathrm{ppm})$ & $200 \pm 20$ & $54 \pm 3$ & $110 \pm 14$ & $41 \pm 2$ \\
\hline $\mathrm{Pt}(\mathrm{ppm})$ & & $55 \pm 3$ & $300 \pm 50$ & $66 \pm 9$ \\
\hline $\operatorname{Re}(\mathrm{ppm})$ & & $54 \pm 7$ & $320 \pm 30$ & $100 \pm 17$ \\
\hline $\mathrm{Rh}(\mathrm{ppm})$ & & $0.64 \pm 0.11$ & $1.0 \pm 0.1$ & $0.75 \pm 0.12$ \\
\hline $\mathrm{Ru}(\mathrm{ppm})$ & & $78 \pm 4$ & $380 \pm 50$ & $107 \pm 4$ \\
\hline $\mathrm{Sb}(\mathrm{ppm})$ & $97 \pm 9$ & $36 \pm 2$ & $32 \pm 4$ & $16 \pm 1$ \\
\hline Sn (ppm) & $210 \pm 20$ & $39 \pm 2$ & $43 \pm 4$ & $23 \pm 3$ \\
\hline $\mathrm{W}(\mathrm{ppm})$ & & $46 \pm 3$ & $250 \pm 20$ & $54 \pm 9$ \\
\hline \multicolumn{5}{|c|}{ Partition coefficients } \\
\hline $\mathrm{D}(\mathrm{Ni})$ & $1.47 \pm 0.05$ & $1.25 \pm 0.03$ & $1.17 \pm 0.03$ & $1.06 \pm 0.02$ \\
\hline $\mathrm{D}(\mathrm{Ag})$ & $0.010 \pm 0.003$ & $0.025 \pm 0.009$ & $0.045 \pm 0.014$ & $0.06 \pm 0.03$ \\
\hline $\mathrm{D}(\mathrm{As})$ & $1.2 \pm 0.1$ & $0.48 \pm 0.09$ & $0.29 \pm 0.05$ & $0.26 \pm 0.07$ \\
\hline $\mathrm{D}(\mathrm{Au})$ & $2.6 \pm 0.6$ & $0.9 \pm 0.2$ & $0.60 \pm 0.12$ & $0.46 \pm 0.08$ \\
\hline $\mathrm{D}(\mathrm{Bi})$ & & $0.0011 \pm 0.0005$ & $0.0013 \pm 0.0004$ & $0.0021 \pm 0.0007$ \\
\hline
\end{tabular}


Table 3. Continued. Composition and partitioning data for experiments with 1:1 Fe:Ni ratios.

\begin{tabular}{lcccc}
\hline Run no. & DD2 & DD6 & DD3 & DD5 \\
\hline $\mathrm{D}(\mathrm{Co})$ & $2.6 \pm 0.3$ & $1.9 \pm 0.2$ & $1.6 \pm 0.1$ & $1.4 \pm 0.2$ \\
$\mathrm{D}(\mathrm{Cu})$ & $0.39 \pm 0.04$ & $0.47 \pm 0.11$ & $0.56 \pm 0.07$ & $0.56 \pm 0.10$ \\
$\mathrm{D}(\mathrm{Ga})$ & & $3.2 \pm 0.9$ & $1.8 \pm 0.3$ & $1.2 \pm 0.2$ \\
$\mathrm{D}(\mathrm{Ge})$ & & $3.0 \pm 0.8$ & $1.7 \pm 0.3$ & $1.1 \pm 0.2$ \\
$\mathrm{D}(\mathrm{Ir})$ & $25 \pm 8$ & $1.5 \pm 0.2$ & $1.4 \pm 0.2$ & $5 \pm 2$ \\
$\mathrm{D}(\mathrm{Mo})$ & $2.3 \pm 0.3$ & $35 \pm 7$ & $13 \pm 5$ & $1.0 \pm 0.2$ \\
$\mathrm{D}(\mathrm{Os})$ & & $0.0023 \pm 0.0009$ & $0.0037 \pm 0.0012$ & $7 \pm 3$ \\
$\mathrm{D}(\mathrm{Pb})$ & $1.0 \pm 0.2$ & $0.73 \pm 0.09$ & $0.006 \pm 0.003$ \\
$\mathrm{D}(\mathrm{Pd})$ & $1.8 \pm 0.4$ & $9 \pm 3$ & $4.5 \pm 1.4$ & $0.69 \pm 0.06$ \\
$\mathrm{D}(\mathrm{Pt})$ & & $27 \pm 7$ & $12 \pm 4$ & $2.4 \pm 0.6$ \\
$\mathrm{D}(\mathrm{Re})$ & & $5 \pm 2$ & $2.9 \pm 1.0$ & $6 \pm 2$ \\
$\mathrm{D}(\mathrm{Rh})$ & & $8.5 \pm 2$ & $2.2 \pm 0.7$ \\
$\mathrm{D}(\mathrm{Ru})$ & & $0.12 \pm 0.03$ & $0.10 \pm 0.03$ & $2.8 \pm 0.7$ \\
$\mathrm{D}(\mathrm{Sb})$ & $0.30 \pm 0.06$ & $0.19 \pm 0.03$ & $0.14 \pm 0.03$ & $0.09 \pm 0.03$ \\
$\mathrm{D}(\mathrm{Sn})$ & & $5.5 \pm 1.3$ & $0.12 \pm 0.04$ \\
$\mathrm{D}(\mathrm{W})$ & & $11 \pm 2$ & & $3.2 \pm 1.1$
\end{tabular}

Data for $\mathrm{Fe}, \mathrm{Ni}$, and $\mathrm{S}$ are from electron microprobe analysis. All other data are from LA ICP-MS.

Errors are $\pm 2 \sigma$ of the mean.

averaged to determine the liquid metal composition. Chabot and Drake (1997) demonstrated that such a method for determining the bulk composition of the metallic liquid was valid by utilizing an independent image analysis technique to determine the $\mathrm{S}$ content of the metallic liquid; the image analysis results showed very good agreement with the $\mathrm{S}$ contents determined by averaging multiple raster beam electron microprobe measurements. Errors for both solid and liquid metal phases were calculated as twice the standard error of the mean.

The concentrations of trace elements were measured by laser ablation inductively coupled plasma mass spectrometry (ICP-MS) microanalysis at the University of Maryland. In situ analyses were carried out using a single-collector ICP-MS (Element 2, Thermo Electron Corp) coupled to a laser ablation system with an output wavelength at $213 \mathrm{~nm}$ (UP213, New Wave Research). The laser was operated with a uniform energy density of $\sim 2.6 \mathrm{~J} / \mathrm{cm}^{2}$. Ablation sampling was done in line scan mode using a spot $30 \mu \mathrm{m}$ in diameter and a $7 \mathrm{~Hz}$ flash rate for the solid metal, and for the liquid metal, a spot $80 \mu \mathrm{m}$ in diameter and a $5 \mathrm{~Hz}$ flash rate. The sample was moved at a rate of $10 \mu \mathrm{m} / \mathrm{s}$ during ablation. The lengths of the line scans varied depending on the specific features of each run product, but were generally 300 to $1000 \mu \mathrm{m}$ in length. Four line scans were conducted in each solid and liquid phase. This analysis approach has proven to be effective for measuring the bulk composition of the quenched metallic liquid in similar previous experimental samples (Chabot et al. 2003). Data were collected for the following masses: ${ }^{57} \mathrm{Fe}$, ${ }^{59} \mathrm{Co},{ }^{62} \mathrm{Ni},{ }^{63} \mathrm{Cu},{ }^{65} \mathrm{Cu},{ }^{69} \mathrm{Ga},{ }^{71} \mathrm{Ga},{ }^{73} \mathrm{Ge},{ }^{75} \mathrm{As},{ }^{95} \mathrm{Mo},{ }^{97} \mathrm{Mo}$, ${ }^{99} \mathrm{Ru},{ }^{101} \mathrm{Ru},{ }^{103} \mathrm{Rh},{ }^{105} \mathrm{Pd},{ }^{107} \mathrm{Ag},{ }^{109} \mathrm{Ag},{ }^{117} \mathrm{Sn},{ }^{118} \mathrm{Sn},{ }^{121} \mathrm{Sb}$, ${ }^{123} \mathrm{Sb},{ }^{182} \mathrm{~W},{ }^{183} \mathrm{~W},{ }^{185} \mathrm{Re},{ }^{188} \mathrm{Os},{ }^{189} \mathrm{Os},{ }^{191} \mathrm{Ir},{ }^{193} \mathrm{Ir},{ }^{194} \mathrm{Pt},{ }^{195} \mathrm{Pt}$, ${ }^{197} \mathrm{Au},{ }^{206} \mathrm{~Pb},{ }^{208} \mathrm{~Pb}$, and ${ }^{209} \mathrm{Bi}$. Analyses of solid and liquid metal pairs were accompanied by the analyses of two standard reference materials (SRM Filomena and SRM 610) before and after the sample acquisition, which provided the calibration curves for determining element concentrations and for constraining instrument drift. Data were processed using the LAMTRACE (Achterbergh et al. 2001) software program, which determines element concentrations based on ratios of count rates for samples and standards, known concentrations in the standards, and the known concentration of an internal standard in the unknowns. Errors reported in Tables 1-3 are twice the standard error of the mean. In a given experiment, if the measurement for an element was below the detection limit (defined as background plus three standard deviations of the background) or if three times the error was greater than $100 \%$ error, then data for that element was not reported for that specific experiment.

\section{PARTITIONING RESULTS}

Solid metal/liquid metal partition coefficients, D, are calculated as the weight concentration of an element in the solid metal divided by the weight concentration of an element in the liquid metal. Calculated partition coefficients are given in Tables 1-3 with errors determined from twice the standard error of the mean for both solid and liquid phases.

Figure 3 plots the partitioning results for $\mathrm{D}(\mathrm{Ni})$, a major element in the experiments. The data from previous experimental studies comes from numerous sources, the references for which are detailed in the appendix (Table A1). The data in Fig. 3 are plotted against the $\mathrm{S}$ content in the metallic liquid, which is known to have a significant effect on solid metal/liquid metal partitioning behavior (e.g., Jones and Drake 1983). To facilitate comparisons that expose effects due to Ni, we have chosen to break all experimental data, both previous and our new results, into three main categories: 
1. Data from the Fe-Ni-S system with $\mathrm{Ni}<20 \%$ in the solid metal; this category encompasses the large majority of previous data, conducted in low-Ni systems.

2. Data from the Fe-Ni-S system with $\mathrm{Ni}>20 \%$ in the solid metal; this grouping includes our experiments with 1:1 Fe:Ni ratios and are the compositions most directly applicable to high-Ni iron meteorites.

3. Data from the Fe-free Ni-S system, representing an extreme end-member in partitioning behavior.

The concentration of $\mathrm{Ni}$ in the solid metal (rather than the liquid metal) was chosen as the discriminating factor since most iron meteorites are believed to be samples of crystallized solid metal (e.g., Scott 1972).

As seen on Fig. 3, our determination of $\mathrm{D}(\mathrm{Ni})$ in the Fe$\mathrm{Ni}-\mathrm{S}$ system with $<20 \% \mathrm{Ni}$ in the solid metal is in good agreement with similar previous data, as would be expected. In the $\mathrm{Ni}-\mathrm{S}$ system, $\mathrm{D}(\mathrm{Ni})$ deviates slightly from the low-Ni previous results; at $\mathrm{S}$ contents $<15 \mathrm{wt} \%, \mathrm{D}(\mathrm{Ni})$ values in the $\mathrm{Ni}-\mathrm{S}$ system and in the Ni-rich $\mathrm{Fe}-\mathrm{Ni}-\mathrm{S}$ system are elevated slightly relative to the low-Ni data. However, overall, these results suggest that the $\mathrm{Ni}$ content has a minimal effect on the value of $\mathrm{D}(\mathrm{Ni})$, even when comparing partitioning behaviors between the extreme end-member Ni-S and low Ni, Fe-Ni-S systems.

Figure 4 shows partitioning results for 20 trace elements contained in our experiments. The symbols used in Fig. 4 are the same as in Fig. 3, with the data fundamentally divided into low-Ni, high-Ni, and $\mathrm{Fe}$-free groups. Table A1 provides the references for the previous data that are plotted on Fig. 4. For the two experiments (\#SS1 and \#SS2) that we conducted in the Fe-Ni-S system with Ni contents $<20 \%$ in the solid metal, our experimental partitioning results are in very good agreement with the available previous experimental data from low-Ni systems. This agreement is expected. The good agreement also gives confidence in our experimental and analytical methods and that discrepancies between our high$\mathrm{Ni}$ data and previous data are due to effects from $\mathrm{Ni}$.

In Fig. 4, it is perhaps easiest to begin by examining the results from the Fe-free Ni-S system, as compared to the lowNi predominantly Fe-S system. These two systems represent end-member compositions, producing the most extreme partitioning differences due to Ni. Many of the trace elements in Fig. 4 show a similar behavior: in the Ni-S system, the partition coefficients increase more quickly with high $\mathrm{S}$ content in the metallic liquid than those in the predominantly $\mathrm{Fe}-\mathrm{S}$ system. Prime examples of this behavior are seen in the elements Ir, Re, and Os as well as Mo. At about $20 \mathrm{wt} \% \mathrm{~S}$, the partition coefficients for these elements are over an order of magnitude greater in the Ni-S system than in the Fe-S system. Similar, though less extreme, behavior is exhibited by many elements, such as $\mathrm{Co}, \mathrm{Ga}, \mathrm{Ge}$, and Pt, plotted in Fig. 4.

In contrast, other elements show little to no change in their solid metal/liquid metal partition coefficients between the Fe-S and Ni-S systems. The partitioning behaviors of As

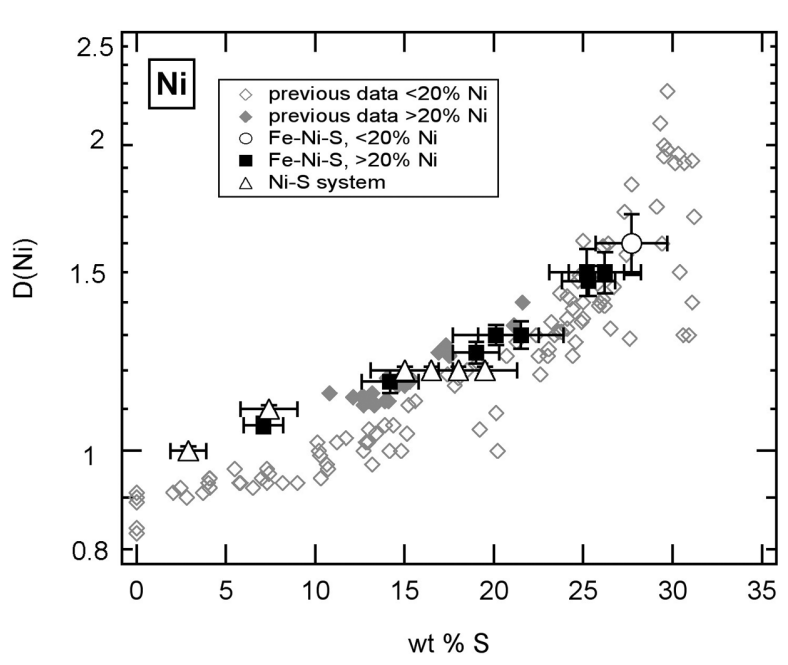

Fig. 3. The solid metal/liquid metal partition coefficient for $\mathrm{Ni}$, $\mathrm{D}(\mathrm{Ni})$, is plotted as a function of the $\mathrm{S}$ content of the metallic liquid. Both previous and our new experimental partitioning results are divided into three main categories for comparison purposes: 1) Low$\mathrm{Ni}$ systems, with $<20 \% \mathrm{Ni}$ in the solid metal. 2) High-Ni systems, with $>20 \%$ Ni in the solid metal. 3) Fe-free Ni-S system. Partitioning behavior in all three systems is observed to be very similar, regardless of the large differences in Ni contents.

and $\mathrm{Au}$ are examples of general indifference to the Fe versus $\mathrm{Ni}$ content of the S-bearing system, as shown in Fig. 4. The elements of $\mathrm{Rh}$ and $\mathrm{Ru}$ also do not appear to show much of an effect of $\mathrm{Ni}$ on their partition coefficients, though data from the low-Ni system are sparse in both of these cases.

In Fig. 4, $\mathrm{Cu}$ exhibits a unique change between solid metal/liquid metal partitioning in the Fe-S and Ni-S systems. In the $\mathrm{Fe}-\mathrm{S}$ system, $\mathrm{Cu}$ is observed to be chalcophile, Sloving, with $\mathrm{D}(\mathrm{Cu})$ decreasing as the $\mathrm{S}$ content of the metallic liquid increases. In contrast, in the Ni-S system, $\mathrm{Cu}$ no longer behaves as a chalcophile element, with $\mathrm{D}(\mathrm{Cu})$ values staying nearly constant over a range of varying $\mathrm{S}$ concentrations. Unlike $\mathrm{Cu}, \mathrm{Ag}$, another chalcophile element shown on Fig. 4, exhibits chalcophile behavior in both the Fe-S and Ni-S systems. Data for $\mathrm{Bi}$ and $\mathrm{Pb}$ in the $\mathrm{Fe}-\mathrm{S}$ system are not available, though both behave as chalcophile elements in the $\mathrm{Ni}-\mathrm{S}$ system. What controls the chalcophile nature of these elements is currently unclear, but interpreting this new data offers potential insight into the factors influencing partitioning behaviors in metallic systems. It is clear that light element content of the metallic liquid alone cannot account for the drastic change in the partitioning behavior of $\mathrm{Cu}$.

Also plotted in Fig. 4 are experiments that contain high $\mathrm{Ni}$ contents but are still in the Fe-Ni-S system. These experiments have $\mathrm{Fe}: \mathrm{Ni}$ ratios that are up to $1: 1$, which is consistent with the compositions observed in the highest $\mathrm{Ni}$ iron meteorites. As one would expect, these data with intermediate Ni contents largely fall between the end-member $\mathrm{Ni}-\mathrm{S}$ and Fe-S data sets. However, an important observation is that the partition coefficients from the 1:1 Fe:Ni experiments 

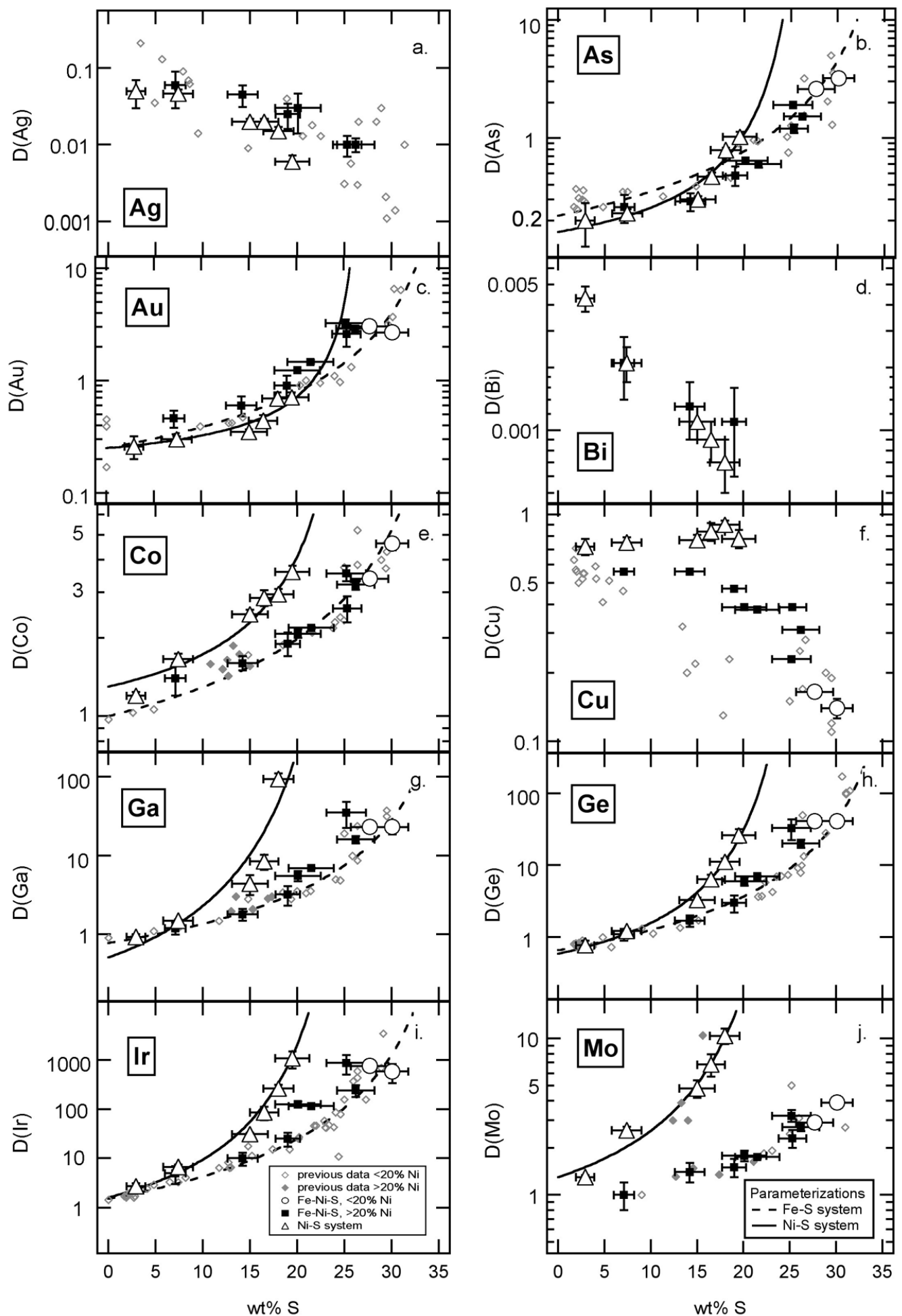

Fig. 4. The solid metal/liquid metal partition coefficients for (a) Ag, (b) As, (c) Au, (d) Bi, (e) Co, (f) Cu, (g) Ga, (h) Ge, (i) Ir, and (j) Mo are plotted as a function of the $\mathrm{S}$ content of the metallic liquid. Both previous and our new experimental partitioning results are divided into three main categories for comparison purposes: 1) Low-Ni systems, with $<20 \% \mathrm{Ni}$ in the solid metal; 2) high-Ni systems, with $20 \% \mathrm{Ni}$ in the solid metal; and 3) Fe-free Ni-S system. The amount of $\mathrm{Ni}$ in the metallic system can affect the partitioning behaviors, with different trace elements showing varying effects. Note that the different graphs have different scales for the y-axes. Also shown on the graphs are best fits of the parameterizations for the partition coefficients in the $\mathrm{Fe}-\mathrm{S}$ and Ni-S systems, with values for the parameterization constants for each element given in Table 4. 

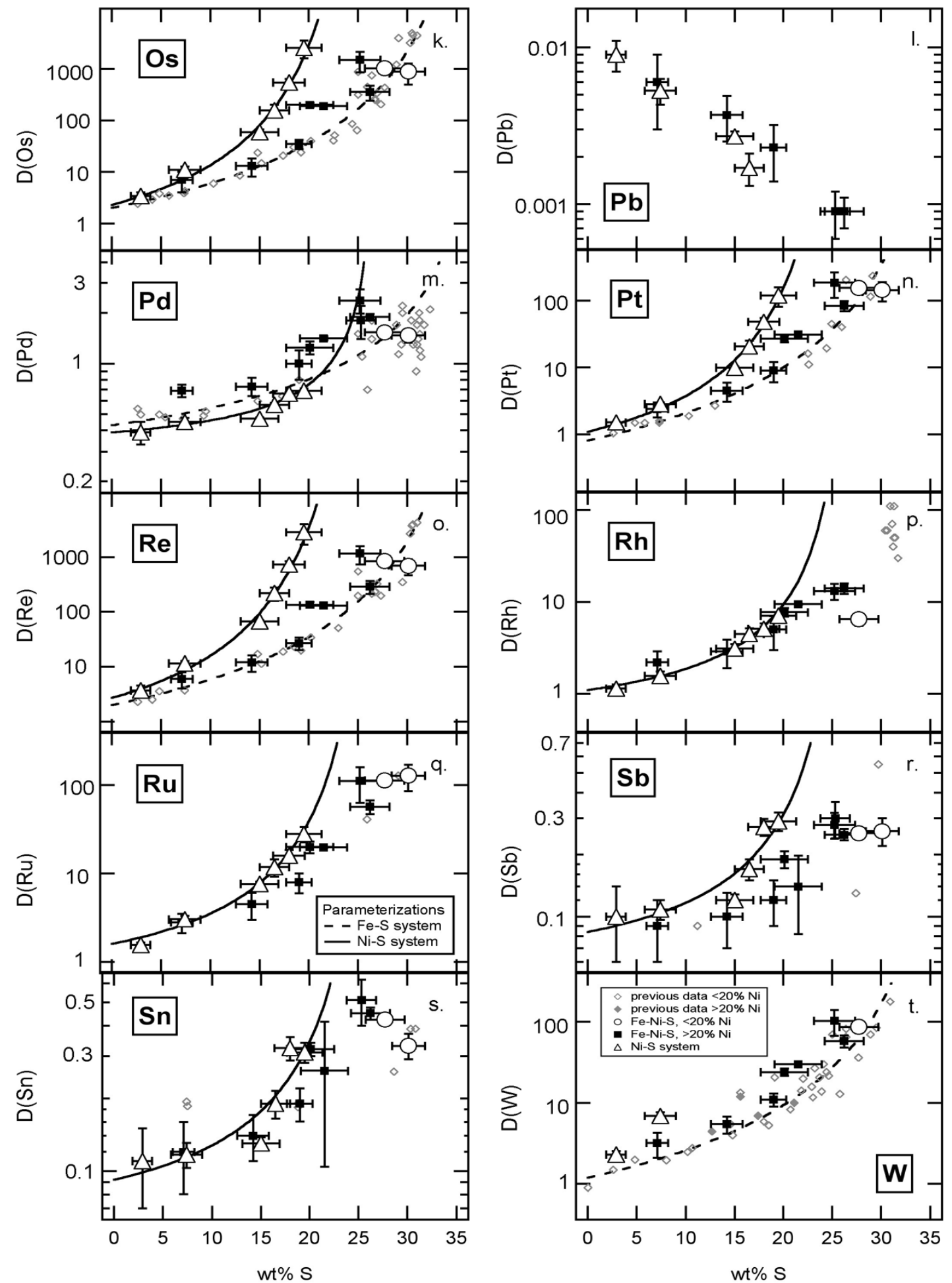

Fig. 4. Continued. The solid metal/liquid metal partition coefficients for (k) Os, (l) Pb, (m) Pd, (n) Pt, (o) Re, (p) Rh, (q) Ru, (r) Sb, (s) Sn, and $(\mathrm{t}) \mathrm{W}$ are plotted as a function of the $\mathrm{S}$ content of the metallic liquid. 
Table 4. Values for the parameterization constants.

\begin{tabular}{lllllc}
\hline & \multicolumn{2}{c}{ Fe-S system $^{\mathrm{a}}$} & & \multicolumn{2}{c}{ Ni-S system } \\
\cline { 2 - 3 } \cline { 5 - 6 } Element & $\mathrm{D}_{\mathrm{o}}$ & $\beta$ & & $\mathrm{D}_{\mathrm{o}}$ & $\beta$ \\
\hline $\mathrm{As}$ & 0.22 & 2.2 & & 0.16 & 3.5 \\
$\mathrm{Au}$ & 0.25 & 2.0 & & 0.25 & 1.9 \\
$\mathrm{Co}$ & 1.0 & 1.2 & & 1.3 & 2.1 \\
$\mathrm{Ga}$ & 0.78 & 2.6 & & 0.51 & 11 \\
$\mathrm{Ge}$ & 0.66 & 3.0 & & 0.59 & 7.2 \\
$\mathrm{Ir}$ & 1.5 & 4.9 & & 1.6 & 13 \\
$\mathrm{Mo}$ & & & & 1.3 & 5.0 \\
$\mathrm{Os}$ & 2.0 & 5.1 & & 2.3 & 13 \\
$\mathrm{Pd}$ & 0.43 & 1.1 & & 0.39 & 1.2 \\
$\mathrm{Pt}$ & 0.81 & 4.4 & & 1.1 & 9.0 \\
$\mathrm{Re}$ & 2.0 & 5.0 & & 2.7 & 13 \\
$\mathrm{Rh}$ & & & & 1.1 & 3.9 \\
$\mathrm{Ru}$ & & & & 1.6 & 5.8 \\
$\mathrm{Sb}$ & & & & 0.084 & 2.4 \\
$\mathrm{Sn}$ & & & & 0.092 & 2.4 \\
$\mathrm{~W}$ & 1.2 & 3.6 & & \\
\hline
\end{tabular}

${ }^{a}$ Values from Chabot and Jones (2003).

do not fall an equal distance between the end-member systems but rather in most cases more closely resemble the partitioning values measured in the $\mathrm{Fe}-\mathrm{S}$ system. The implications of this observation to iron meteorites is discussed in the next section.

Conceptual models that envision the metallic liquid as being composed of "metal domains" and "nonmetal domains" have had success at explaining partitioning behavior in solid metal/liquid metal systems. Based on this concept, Jones and Malvin (1990) introduced a quantitative expression for parameterizing solid metal/liquid metal partition coefficients as a function of the light element present in the system, such as S, P, or C. Subsequently, Chabot and Jones (2003) modified the mathematical expression, though the basic concept of different "domains" in the metallic liquid that controlled partitioning behavior was maintained. Siderophile elements strongly prefer the metal domains to the nonmetal domains in the metallic liquid, and the solid metal/liquid metal partition coefficient for these siderophile elements can be expressed as a function of the concentration of available metal domains:

$$
\frac{1}{D}=\frac{(\text { metal domains })^{\beta}}{D_{O}}
$$

This expression is Equation 5 from Chabot and Jones (2003). $D$ is the solid metal/liquid metal partition coefficient, $D_{o}$ is the partition coefficient in the non-metal-free system, and $\beta$ is a constant specific to the element being parameterized. The variable of metal domains refers to the fraction of the domains in the metallic liquid that are nonmetal free; the fraction of metal domains and the fraction of nonmetal domains add up to one.

For partitioning in the Fe-S system, the metallic liquid is envisioned as being composed of nonmetal $\mathrm{FeS}$ domains and metal Fe domains; this is consistent with the Fe-S phase

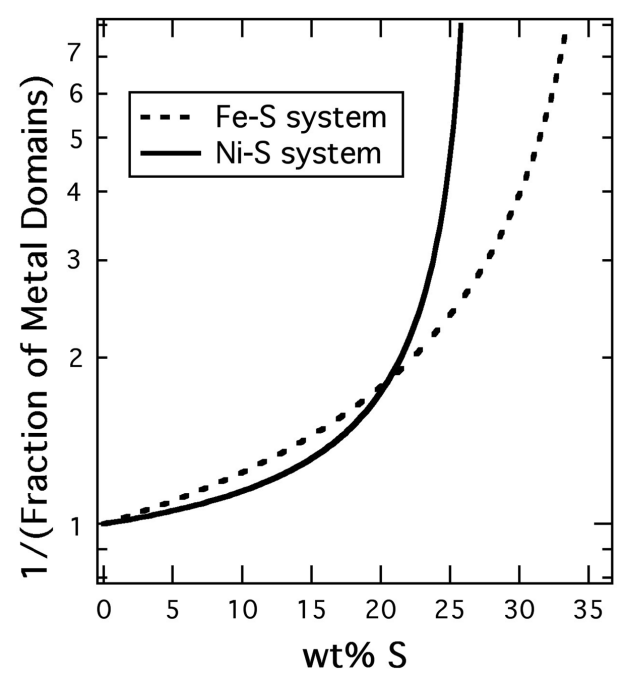

Fig. 5. The metallic liquid is envisioned as being composed of "metal domains" and "nonmetal domains." In the Fe-S system, there are Fe metal domains and $\mathrm{FeS}$ domains, but in the $\mathrm{Ni}-\mathrm{S}$ system, there are $\mathrm{Ni}$ metal domains and $\mathrm{Ni}_{3} \mathrm{~S}_{2}$ domains. The difference in speciation between the Fe-S and Ni-S systems results in slightly different functional forms for the fraction of metal domains available at a given $\mathrm{S}$ content, which influences the solid metal/liquid metal partitioning behavior of siderophile elements, as shown on Fig. 4.

diagram, which has an Fe-FeS eutectic at about $31 \mathrm{wt} \% \mathrm{~S}$ (Massalski et al. 1990). In this system, the fraction of metal $\mathrm{Fe}$ domains is calculated as a function of the mole concentration of $\mathrm{S}$ in the metallic liquid, $X_{S}$, as:

$$
\text { Fe metal domains }=\frac{\left(1-2 X_{S}\right)}{\left(1-X_{S}\right)}
$$

In the Ni-S system, a slightly different speciation of $\mathrm{S}$ in the metallic liquid may occur. The Ni-S phase diagram shows

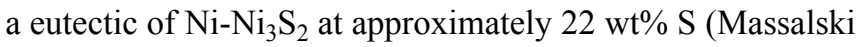
et al. 1990). Thus, the metallic liquid can be envisioned as being composed of metal $\mathrm{Ni}$ domains and nonmetal $\mathrm{Ni}_{3} \mathrm{~S}_{2}$ domains. The fraction of metal Ni domains as a function of the $\mathrm{S}$ content of the metallic liquid is given as:

$$
\text { Ni metal domains }=\frac{\left(1-2.5 X_{S}\right)}{\left(1-2 X_{S}\right)}
$$

Figure 5 illustrates how the different speciation in the metallic liquid affects the fraction of available metal domains in the $\mathrm{Fe}-\mathrm{S}$ and $\mathrm{Ni}-\mathrm{S}$ systems. There are some general similarities between the two functional forms given in Fig. 5 and the experimental partitioning data shown on Fig. 4 for many siderophile elements. Most notably, our experimental data show that partition coefficients frequently achieve higher values with increasing $\mathrm{S}$ content more quickly in the Ni-S system than in the Fe-S system. This is consistent with Equation 1, where the partition coefficient is an inverse 
function of the fraction of available metal domains; as plotted in Fig. 5, the fraction of metal domains decreases more rapidly in the Ni-S system than in the Fe-S system with increasing $\mathrm{S}$ content of the metallic liquid, due to the different speciation of $\mathrm{S}$ in the two systems.

Using Equations 1 and 3, we determined best fits to our new partitioning data in the Ni-S system. The calculated values for the parameterization constants of $D_{o}$ and $\beta$ for each element in the Ni-S system are given in Table 4, along with the values for these constants in the Fe-S system as previously determined by Chabot and Jones (2003). The best-fit parameterizations in the Ni-S and $\mathrm{Fe}-\mathrm{S}$ systems are also shown in Fig. 4. The different speciation of the metallic liquids in the Fe-S and Ni-S systems does a very good job at explaining the observed partitioning behaviors for siderophile elements in these two systems. Parameterizations have not yet been developed for the partitioning of chalcophile elements (such as $\mathrm{Ag}, \mathrm{Bi}, \mathrm{Cu}$, and $\mathrm{Pb}$ in Fig. 4) in either the $\mathrm{Fe}-\mathrm{S}$ or Ni-S systems; as with the siderophile elements, accounting for the different speciation of the metallic liquid in Fe-S and Ni-S systems will likely be important when parameterizing chalcophile behavior.

\section{IMPLICATIONS FOR IRON METEORITES}

Our experimental results demonstrate that Ni content can have a large effect on solid metal/liquid metal partitioning in the Fe-Ni-S system. Comparing partitioning in the Ni-S and (predominantly) Fe-S systems, the maximum difference in partitioning behaviors for the two systems occurs at about $20 \mathrm{wt} \% \mathrm{~S}$, as shown in Fig. 4. Figure 6a summarizes this maximum difference between the two end-member systems by plotting the ratio of the partition coefficient in the Ni-S system to the partition coefficient in the Fe-S system at $20 \mathrm{wt} \% \mathrm{~S}$. To do this, experiment \#NN4 was compared to an estimated value for the low-Ni system, as indicated by the trend of the previous experimental data. For Ga and Mo, which were not measured in experiment \#NN4, the values from experiment \#NN5 were used, which contained $18 \mathrm{wt} \% \mathrm{~S}$ in the metallic liquid. Errors were estimated by determining the percentage error for the partition coefficient from experiment \#NN4 and multiplying that percentage by a factor of the square root of two; this is based on the assumption that the error in the estimation of the low-Ni partitioning value is similar to the error of experiment \#NN4 and that these errors are independent. These errors are simple estimates and provide some indication of the uncertainties in the values.

Some elements, such as the highly siderophile element pair Re and Os, have a difference of nearly two orders of magnitude between their partition coefficients in the Fe-S and Ni-S systems, as shown on Fig. 6a. Other elements, such as $\mathrm{Pd}, \mathrm{Au}$, and $\mathrm{Ni}$, have essentially no change in their partition coefficients at $20 \mathrm{wt} \% \mathrm{~S}$ between partitioning in the Ni-S and $\mathrm{Fe}-\mathrm{S}$ systems and consequently plot near a ratio of one on

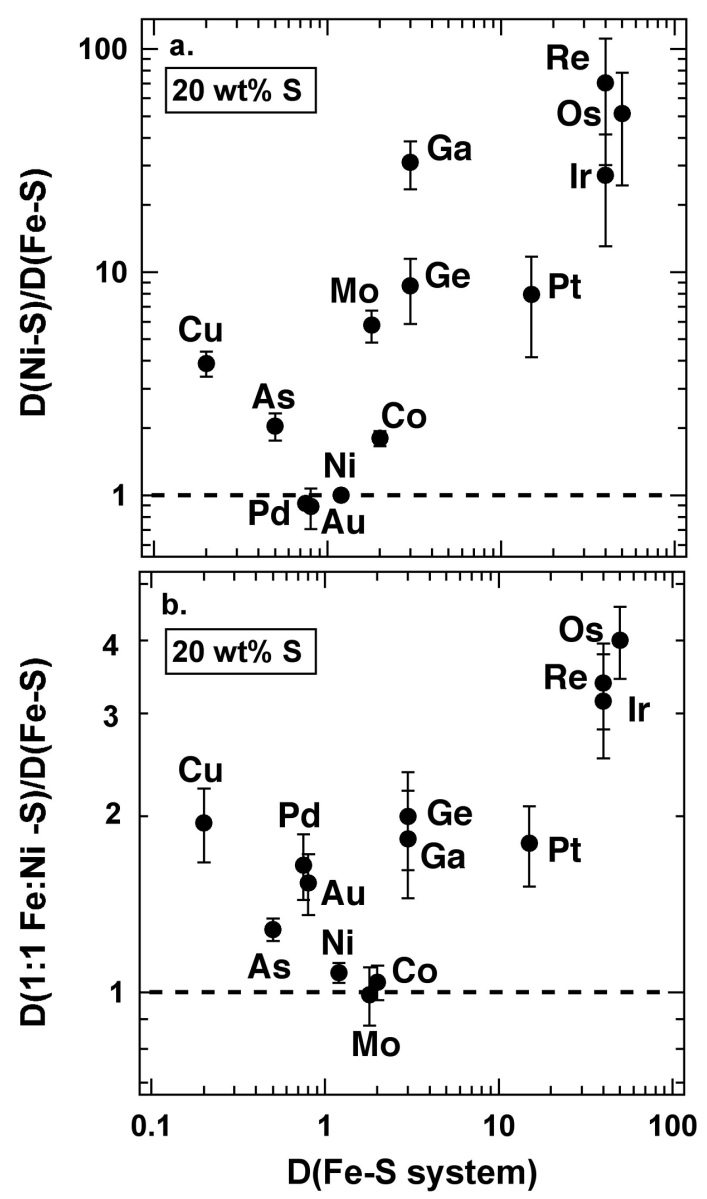

Fig. 6. (a) For many elements, the largest difference in partitioning behavior occurs when the metallic liquid has about $20 \mathrm{wt} \% \mathrm{~S}$, as seen on Fig. 4. For a metallic liquid with $20 \mathrm{wt} \% \mathrm{~S}$, the ratio of an element's partition coefficient in the Ni-S system to its partition coefficient in the predominantly Fe-S system is plotted as a function of the element's partition coefficient in the Fe-S system. If an element showed identical partitioning behaviors in the Ni-S and Fe-S systems, it would have a ratio of one on this plot, indicated with a dashed line. In contrast, some elements have partition coefficients that are over an order of magnitude higher in the Ni-S system. (b) A similar plot shows the ratio of partition coefficients between a system with equal parts $\mathrm{Fe}$ and $\mathrm{Ni}(1: 1 \mathrm{Fe}: \mathrm{Ni})$ and the Fe-S system. In this system, which is relevant to high $\mathrm{Ni}$ iron meteorites, the partition coefficients for most elements show a factor of two or less variation due to the different $\mathrm{Ni}$ contents of the two systems.

Fig. 6a. Figure 6a shows a general correlation between the value of an element's solid metal/liquid metal partition coefficient and the effect of $\mathrm{Ni}$ on that partition coefficient; elements with larger solid metal/liquid metal partition coefficients are more affected by the Ni content of the system.

Figure $6 \mathrm{a}$ illustrates that $\mathrm{Ni}$ content can significantly influence solid metal/liquid metal partitioning behavior, and that partition coefficients determined in low-Ni Fe-(Ni)-S systems would not be appropriate to apply to partitioning in the Ni-S system for some elements. However, even the most Ni-rich iron meteorites did not crystallize in the Ni-S system 
but rather in a system with about equal parts of $\mathrm{Fe}$ and Ni. Figure $6 \mathrm{~b}$ is similar to Fig. $6 \mathrm{a}$, but plots the ratio of an element's partition coefficient in a system with $1: 1 \mathrm{Fe}: \mathrm{Ni}$ concentrations to the partition coefficient in the low-Ni, dominantly Fe-S system. Similarly to Fig. 6a, experiment \#SS5 was used to produce Fig. 6b. As shown in Fig. 6b, even the most influenced elements show only a factor of four change in their partition coefficients between the two systems; the large majority of elements have only a factor of two or less variation in their partition coefficients. Additionally, Fig. $6 \mathrm{~b}$ summarizes the difference between partitioning in the two systems at $20 \mathrm{wt} \% \mathrm{~S}$, which is the $\mathrm{S}$ content which shows the largest difference between the Ni-S and Fe-S systems. At lower S contents, differences between $\mathrm{Ni}$-rich and Ni-poor systems are even smaller.

There are some intriguing observations that can be made by comparing Fig. $6 \mathrm{a}$ and $6 \mathrm{~b}$. For one, $\mathrm{Au}$ and $\mathrm{Pd}$ appear to have similar partition coefficients in the end-member Fe-S and Ni-S systems, but at the intermediate 1:1 Fe:Ni-S system, both $\mathrm{D}(\mathrm{Au})$ and $\mathrm{D}(\mathrm{Pd})$ have elevated values. Examining the full data sets in Fig. $4 \mathrm{c}$ and $4 \mathrm{~m}$ seem to further support this confusing observation. It would be natural to predict that the behavior in the intermediate system would fall between the end-member systems, unlike what is observed here for $\mathrm{D}(\mathrm{Au})$ and $\mathrm{D}(\mathrm{Pd})$. Though we have no explanation for this observation, we do note that the discrepancy between the different systems is minor, amounting to less than a factor of two.

Our motivation for this project was to determine if the $\mathrm{Ni}$ content of a crystallizing metallic melt influenced the resulting trace element concentrations recorded in iron meteorites. Our experimental results indicate that for compositions relevant to iron meteorites, the influence of $\mathrm{Ni}$ on elemental partitioning behavior during iron meteorite crystallization would have been minor in most situations. Though some highly siderophile elements such as Ir, Os, and Re exhibit up to factor of four increases in their partition coefficients, such increases are still minor effects when compared to those of other influences, such as the S content of the metallic melt. This point is illustrated on Fig. 4, which shows the effects of both S and Ni. Experiments conducted in the 1:1 Fe:Ni-S system may fall slightly outside the scatter of the previous, low-Ni experiments, but the offset is small in comparison to the overall partitioning behavior trends due to the presence of $\mathrm{S}$. There is the possibility that in some cases, if an iron meteorite crystallized in a high-Ni system with a $\mathrm{S}$ content that was extremely stable, that the effects of $\mathrm{Ni}$ could become important influences on elemental partitioning behavior and consequently the resulting iron meteorite composition. Perhaps the best chance for such circumstances could be for some members of the nonmagmatic IAB iron meteorite group, a group that has many high Ni members but whose formation history appears more complex than fractional crystallization as experienced by the magmatic groups (e.g., Benedix et al. 2000; Wasson and Kallemeyn 2002). In any system with a low $S$ content (such as IVB irons) (e.g., Chabot 2004), the influence of $\mathrm{Ni}$ on partitioning behavior is small, as seen in Fig. 4.

However, for most situations involving $\mathrm{S}$ as a major element, including the effect of $\mathrm{Ni}$ on partitioning behavior in iron meteorite crystallization models will lead to only minor differences in the model calculations. For iron meteorites crystallizing in the presence of an evolving $\mathrm{S}$ bearing metallic melt, we conclude that models of iron meteorite crystallization can safely neglect the effect of $\mathrm{Ni}$ when modeling the entire range of $\mathrm{Ni}$ contents measured in iron meteorites.

Acknowledgments-First, we very much appreciate the review by $\mathrm{A}$. Kracher, which pointed out that the speciation of $\mathrm{S}$ in a $\mathrm{Ni}-\mathrm{S}$ metallic liquid could be $\mathrm{Ni}_{3} \mathrm{~S}_{2}$, as compared to $\mathrm{FeS}$ in the $\mathrm{Fe}-\mathrm{S}$ system. This suggestion offered a simple explanation for many of the partitioning differences observed in our data between the Ni-S and $\mathrm{Fe}-\mathrm{S}$ systems, and including this explanation in turn greatly strengthened the final paper. Reviews by $\mathrm{H}$. Watson and D. Mittlefehldt, as well as comments from associate editor C. Floss, were also very useful in improving and clarifying the final paper. This work was supported by the APL Mentorship Program and NASA grants NNG06GI13G to N. L. C., NNG04GG17G to W. F. M., and NNG06GF56G to T. J. M.

\section{Editorial Handling-Dr. Christine Floss}

\section{REFERENCES}

Achterbergh E. V., Ryan C. G., Jackson S. E., and Griffin W. L. 2001. Appendix 3: Data reduction software for LA-ICP-MS. In Laser ablation-ICP-MS in the earth sciences, edited by Sylvester P. Mineralogical Association of Canada, Short Course Series, vol. 29. Quebec: Mineralogical Association of Canada. 243 p.

Benedix G. K., McCoy T. J., Keil K., and Love S. G. 2000. A petrologic study of the IAB iron meteorites: Constraints on the formation of the IAB-winonaite parent body. Meteoritics \& Planetary Science 35:1127-1141.

Bild R. W. and Drake M. J. 1978. Experimental investigations of trace element fractionation in iron meteorites. I-Early results. Proceedings, 9th Lunar and Planetary Science Conference. pp. 1407-1421.

Chabot N. L. 2004. Sulfur contents of the parental metallic cores of magmatic iron meteorites. Geochimica et Cosmochimica Acta 68:3607-3618.

Chabot N. L. and Drake M. J. 1997. An experimental study of silver and palladium partitioning between solid and liquid metal, with applications to iron meteorites. Meteoritics \& Planetary Science 32:637-645.

Chabot N. L. and Haack H. 2006. Evolution of asteroidal cores. In Meteorites and the early solar system II, edited by Lauretta D. S. and McSween H. Y. Jr. Tucson, Arizona: The University of Arizona Press. pp. 747-771.

Chabot N. L. and Jones J. H. 2003. The parameterization of solid metal-liquid metal partitioning of siderophile elements. Meteoritics \& Planetary Science 38:1425-1436. 
Chabot N. L., Campbell A. J., Jones J. H., Humayun M., and Agee C. B. 2003. An experimental test of Henry's Law in solid metalliquid metal systems with implications for iron meteorites. Meteoritics \& Planetary Science 38:181-196.

Fleet M. E. and Stone W. E. 1991. Partitioning of platinum-group elements in the Fe-Ni-S system and their fractionation in nature. Geochimica et Cosmochimica Acta 55:245-253.

Fleet M. E., Liu M., and Crocket J. H. 1999. Partitioning of trace amounts of highly siderophile elements in the Fe-Ni-S system and their fractionation in nature. Geochimica et Cosmochimica Acta 63:2611-2622.

Haack H. and McCoy T. J. 2003. Iron and stony-iron meteorites. In Meteorites, comets, and planets, edited by Davis A. M. Treatise on Geochemistry, vol. 1, edited by Holland H. D. and Turekian K. K. Oxford: Elsevier-Pergamon. pp. 325-346.

Haack H. and Scott E. R. D. 1993. Chemical fractionations in group IIIAB iron meteorites: Origin by dendritic crystallization of an asteroidal core. Geochimica et Cosmochimica Acta 57:34573472.

Hillgren V. J. 1993. Partitioning behavior of moderately siderophile elements in Ni-rich systems: Implications for the Earth and Moon. Ph.D. thesis, The University of Arizona, Tucson, Arizona, USA.

Hongsresawat S., Chabot N. L., and Jones J. H. 2002. Modeling the solidification of magmatic iron meteorites using experimental $\mathrm{Cu}$ partitioning (abstract \#1337). 33rd Lunar and Planetary Science Conference. CD-ROM.

Hsieh K.-C., Chang Y. A., and Zhong T. 1982. The Fe-Ni-S system above $700{ }^{\circ} \mathrm{C}$ (iron-nickel-sulfur). Bulletin of Alloy Phase Diagrams 3:165-172.

Jones J. H. and Drake M. J. 1983. Experimental investigations of trace element fractionation in iron meteorites. II: The influence of sulfur. Geochimica et Cosmochimica Acta 47:1199-1209.

Jones J. H. and Drake M. J. 1986. Geochemical constraints on core formation in the Earth. Nature 322:221-228.

Jones J. H. and Malvin D. J. 1990. A nonmetal interaction model for the segregation of trace metals during solidification of $\mathrm{Fe}-\mathrm{Ni}-\mathrm{S}$, Fe-Ni-P, and Fe-Ni-S-P alloys. Metallurgical Transactions B 21: 697-706.

Kracher A., Willis J., and Wasson J. T. 1980. Chemical classification of iron meteorites-IX. A new group (IIF), revision of IAB and IIICD, and data on 57 additional irons. Geochimica et Cosmochimica Acta 44:773-787.

Liu M. and Fleet M. E. 2001. Partitioning of siderophile elements (W, Mo, As, Ag, Ge, Ga, and Sn) and Si in the Fe-S system and their fractionation in iron meteorites. Geochimica et Cosmochimica Acta 65:671-682.

Lod $\overline{d e r s ~ K . ~ a n d ~ P a l m e ~ H . ~ 1991 . ~ O n ~ t h e ~ c h a l c o p h i l e ~ c h a r a c t e r ~ o f ~}$ molybdenum: Determination of sulfide/silicate partition coefficients of Mo and W. Earth and Planetary Science Letters 103:311-324.

Malvin D. J., Wang D., and Wasson J. T. 1984. Chemical classification of iron meteorites-X. Multi-element studies of 43 irons, resolution of group IIIE from IIIAB, and evaluation of $\mathrm{Cu}$ as a taxonomic parameter. Geochimica et Cosmochimica Acta 48:785-804.

Malvin D. J., Jones J. H., and Drake M. J. 1986. Experimental investigations of trace element fractionation in iron meteorites. III: Elemental partitioning in the system Fe-Ni-S-P. Geochimica et Cosmochimica Acta 50:1221-1231.
Massalski T. B., Okamoto H., Subramanian P. R., and Kacprzak L., editors. 1990. Binary alloy phase diagrams, 2nd ed. Materials Park, Ohio: ASM International. 3 vol.

Schaudy R., Wasson J. T., and Buchwald V. F. 1972. The chemical classification of iron meteorites. VI. A re-investigation of irons with $\mathrm{Ge}$ concentrations lower than 1 ppm. Icarus 17:174-192.

Scott E. R. D. 1972. Chemical fractionation in iron meteorites and its interpretation. Geochimica et Cosmochimica Acta 36:12051236 .

Scott E. R. D. 1979. Origin of anomalous iron meteorites. Mineralogical Magazine 43:415-421.

Scott E. R. D. and Wasson J. T. 1975. Classification and properties of iron meteorites. Reviews of Geophysics and Space Physics 13: 527-546.

Scott E. R. D. and Wasson J. T. 1976. Chemical classification of iron meteorites-VIII. Groups IC, IIE, IIIF, and 97 other irons. Geochimica et Cosmochimica Acta 40:103-115.

Scott E. R. D., Wasson J. T., and Buchwald V. F. 1973. The chemical classification of iron meteorites. VII. A re-investigation of iron with Ge concentrations between 25 and $80 \mathrm{ppm}$. Geochimica et Cosmochimica Acta 37:1957-1983.

Wasson J. T. 1967. The chemical classification of iron meteorites: I. A study of iron meteorites with low concentrations of gallium and germanium. Geochimica et Cosmochimica Acta 31:161-180.

Wasson J. T. 1969. The chemical classification of iron meteoritesIII. Hexahedrites and other irons with germanium concentrations between 80 and 200 ppm. Geochimica et Cosmochimica Acta 33: 859-876.

Wasson J. T. 1970. The chemical classification of iron meteorites IV. Irons with Ge concentrations greater than $190 \mathrm{ppm}$ and other meteorites associated with group I. Icarus 12:407-423.

Wasson J. T. 1990. Ungrouped iron meteorites in Antarctica: Origin of anomalously high abundance. Science 249:900-902.

Wasson J. T. and Kallemeyn G. W. 2002. The IAB iron-meteorite complex: A group, five subgroups, numerous grouplets, closely related, mainly formed by crystal segregation in rapidly cooling melts. Geochimica et Cosmochimica Acta 66: 2445-2473.

Wasson J. T. and Kimberlin J. 1967. The chemical classification of iron meteorites-II. Irons and pallasites with germanium concentrations between 8 and 100 ppm. Geochimica et Cosmochimica Acta 31:2065-2093.

Wasson J. T. and Schaudy R. 1971. The chemical classification of iron meteorites - V. Groups IIIC and IIID and other irons with germanium concentrations between 1 and 25 ppm. Icarus 14:5970 .

Wasson J. T., Ouyang X., Wang J., and Jerde E. 1989. Chemical classification of iron meteorites: XI. Multi-element studies of 38 new irons and the high abundance of ungrouped irons from Antarctica. Geochimica et Cosmochimica Acta 53:735-744.

Wasson J. T., Choi B.-G., Jerde E. A., and Ulff-Møller F. 1998. Chemical classification of iron meteorites: XII. New members of the magmatic groups. Geochimica et Cosmochimica Acta 62: $715-724$.

Willis J. and Goldstein J. I. 1982. The effects of C, P, and S on trace element partitioning during solidification in $\mathrm{Fe}-\mathrm{Ni}$ alloys. Proceedings, 13th Lunar and Planetary Science Conference. pp. A435-A445. 


\section{APPENDIX}

Table A1. References for previous experimental solid metal/liquid metal partitioning data with the number of partition coefficients provided by the reference for each element.

\begin{tabular}{|c|c|c|c|c|c|c|c|c|c|c|c|c|c|c|c|c|c|c|c|}
\hline Reference & $\mathrm{Ag}$ & As & $\mathrm{Au}$ & Co & $\mathrm{Cu}$ & $\mathrm{Ga}$ & $\mathrm{Ge}$ & $\mathrm{Ir}$ & Mo & $\mathrm{Ni}$ & Os & $\mathrm{Pd}$ & $\mathrm{Pt}$ & $\operatorname{Re}$ & $\mathrm{Rh}$ & $\mathrm{Ru}$ & $\mathrm{Sb}$ & Sn & $\mathrm{W}$ \\
\hline Bild and Drake (1978) & & & 1 & 1 & & & & & & 4 & & & & & & & & & \\
\hline Chabot and Drake (1997) & 8 & & & & & & & & & & & 11 & & & & & & & \\
\hline Chabot et al. (2003) & 7 & 21 & & 11 & 17 & 9 & 11 & 26 & & 42 & 25 & 11 & 14 & 16 & & & 3 & & 26 \\
\hline Fleet and Stone (1991) & & & & & & & & & & 5 & & 9 & & & 9 & & & & \\
\hline Fleet et al. (1999) & & & 2 & & & & & 2 & & 4 & 9 & 2 & 2 & 7 & & 2 & & & \\
\hline Hillgren (1993) & & & & 8 & & 6 & & & 8 & 23 & & & & & & & & & 5 \\
\hline Hongsresawat et al. (2002) & & & & & 10 & & & & & 10 & & & & & & & & & \\
\hline Jones and Drake (1983) & & & 7 & & & 3 & 16 & 7 & & 27 & & & & & & & & & \\
\hline Jones and Drake (1986) & & & 1 & 1 & & 1 & & 1 & 1 & 1 & & & & 1 & & & & & 1 \\
\hline Jones and Malvin (1990) & & & 5 & 3 & & 1 & 2 & 3 & & 15 & & & & & & & & & \\
\hline Jones et al. (1993) & & & 1 & & & & & & 1 & & & 1 & & & & & & & \\
\hline Liu and Fleet (2001) & 7 & 6 & & & & 6 & 6 & & 4 & & & & & & & & & 7 & 4 \\
\hline Lodders and Palme (1991) & & & & & & & & & 1 & & & & & & & & & & \\
\hline Willis and Goldstein (1982) & & & & & & & 2 & 2 & & 5 & & & & & & & & & \\
\hline
\end{tabular}

